\title{
Suppression of protein tyrosine phosphatase N23 predisposes to breast tumorigenesis via activation of FYN kinase
}

\author{
Siwei Zhang, ${ }^{1,2}$ Gaofeng Fan, ${ }^{1,3}$ Yuan Hao, ${ }^{1}$ Molly Hammell, ${ }^{1}$ John Erby Wilkinson, ${ }^{4}$ \\ and Nicholas K. Tonks ${ }^{1}$ \\ ${ }^{1}$ Cold Spring Harbor Laboratory, Cold Spring Harbor, New York 11724, USA; ${ }^{2}$ Department of Molecular Genetics and \\ Microbiology, Stony Brook University, Stony Brook, New York 11794, USA; ${ }^{3}$ School of Life Science and Technology, ShanghaiTech \\ University, Shanghai, 201210, China; ${ }^{4}$ Unit for Laboratory Animal Medicine, Department of Pathology, University of Michigan, \\ Ann Arbor, Michigan 48109, USA
}

Disruption of the balanced modulation of reversible tyrosine phosphorylation has been implicated in the etiology of various human cancers, including breast cancer. Protein Tyrosine Phosphatase N23 (PTPN23) resides in chromosomal region 3p21.3, which is hemizygously or homozygously lost in some breast cancer patients. In a lossof-function PTPome screen, our laboratory identified PTPN23 as a suppressor of cell motility and invasion in mammary epithelial and breast cancer cells. Now, our TCGA (The Cancer Genome Atlas) database analyses illustrate a correlation between low PTPN23 expression and poor survival in breast cancers of various subtypes.

Therefore, we investigated the tumor-suppressive function of PTPN23 in an orthotopic transplantation mouse model. Suppression of PTPN23 in Comma 1D $\beta$ cells induced breast tumors within 56 wk. In PTPN23-depleted tumors, we detected hyperphosphorylation of the autophosphorylation site tyrosine in the SRC family kinase (SFK) FYN as well as Tyr142 in $\beta$-catenin. We validated the underlying mechanism of PTPN23 function in breast tumorigenesis as that of a key phosphatase that normally suppresses the activity of FYN in two different models. We demonstrated that tumor outgrowth from PTPN23-deficient BT474 cells was suppressed in a xenograft model in vivo upon treatment with AZD0530, an SFK inhibitor. Furthermore, double knockout of FYN and PTPN23 via CRISPR/CAS9 also attenuated tumor outgrowth from PTPN23 knockout Cal51 cells. Overall, this mechanistic analysis of the tumor-suppressive function of PTPN23 in breast cancer supports the identification of FYN as a therapeutic target for breast tumors with heterozygous or homozygous loss of PTPN23.

[Keywords: FYN; PTPN23; breast cancer; tumor suppressor; tyrosine phosphorylation]

Supplemental material is available for this article.

Received July 5, 2017; revised version accepted October 6, 2017.

The dynamic regulation of phosphorylation of tyrosyl residues in proteins is maintained by the synchronized and complementary activity of two enzyme families: protein tyrosine kinases (PTKs) and protein tyrosine phosphatases (PTPs). Disturbance of their functions disrupts the balance of reversible tyrosine phosphorylation, which impacts the normal patterns of intercellular and intracellular signals and has been implicated in the etiology of various human diseases, including cancer. Progress in understanding of the functions of PTKs in cancer has resulted in the development of novel therapeutics that target specific changes in protein tyrosine phosphorylationdependent signaling that drive cancer etiology (Hunter 2009; Sliwkowski and Mellman 2013). In contrast, the therapeutic potential of the PTPs has yet to be exploited

Corresponding author: tonks@cshl.edu

Article published online ahead of print. Article and publication date are online at http://www.genesdev.org/cgi/doi/10.1101/gad.304261.117. fully. Chromosomal aberrations, copy number variations, and mutations have also been characterized in several PTP genes in human cancer; in fact, now there are several examples of PTPs as tumor suppressors or the products of oncogenes (Alonso et al. 2004; Ostman et al. 2006; Julien et al. 2011; Zhao et al. 2015). Nevertheless, the mechanistic definition of the function of the majority of individual PTPs remains to be established.

Breast cancer, which is the most prevalent malignancy in women, is a heterogeneous disease that can be categorized into five subtypes based on expression of particular markers. They are luminal A and B (which are hormone receptor-positive [estrogen and progesterone receptors]),

(C) 2017 Zhang et al. This article is distributed exclusively by Cold Spring Harbor Laboratory Press for the first six months after the full-issue publication date (see http://genesdev.cshlp.org/site/misc/terms.xhtml). After six months, it is available under a Creative Commons License (Attribution-NonCommercial 4.0 International), as described at http://creativecommons.org/ licenses/by-nc/4.0/. 
HER2-positive (which are defined by high levels of the HER2 PTK and are hormone receptor-negative), triplenegative (which is both HER2-negative and hormone receptor-negative and has also been classified as basallike), and normal-like (which is similar to luminal cancer, being hormone receptor-positive but HER2-negative). Currently, prognosis and therapeutic strategy vary for each subtype. A variety of drugs that target HER2 have been developed, including antibodies such as herceptin/ trastuzumab, antibody conjugates such as T-DM1/ Kadcyla, and small-molecule drugs such as lapatinib. Although these target the cancer cell with specificity, there remain problems of resistance, both intrinsic and acquired, that limit the effectiveness of such therapies. In contrast, triple-negative breast cancer (TNBC), which is aggressive and characterized by poor prognosis (Rakha et al. 2008), is treated with combinations of surgery, radiation, and chemotherapies. It has been reported that there are distinct tyrosine phosphorylation-dependent signatures associated with basal breast cancer cells, in particular featuring the SRC family of PTKs (Hochgrafe et al. 2010). Nevertheless, these remain to be exploited for targeted therapy, and further insights into the signaling changes associated with the various breast cancer subtypes are required.

In order to understand the potential therapeutic significance of any alterations in tyrosine phosphorylation associated with breast cancer, it is essential to establish the contribution of both PTKs and PTPs to such changes. Some progress has been made characterizing the function of particular PTPs in breast cancer models. Considering the conventional oncogenic function of PTKs and prompted by their opposing enzymatic function, the PTPs have commonly been considered tumor-suppressive. Studies in cell and animal models have implicated PTPRE, PTPRJ, and PTPN13 in the development of breast cancer (Freiss and Vignon 2004). The expression of PTPRG is down-regulated in breast tumors (Zheng et al. 2000). In mechanistic studies, PTPRO (Yu et al. 2012), PTPN9 (Yuan et al. 2010), and PTPN13 (Zhu et al. 2008) have been shown to antagonize HER2 signaling via direct dephosphorylation in several models of mammary carcinogenesis. Nevertheless, some PTPs may also act as positive regulators in the etiology of particular breast cancer subtypes. PTPRA is overexpressed in $\sim 30 \%$ of primary human breast cancers, with low tumor grade (Ardini et al. 2000), whereas PRL-3 (Radke et al. 2006) and LAR (Levea et al. 2000) are elevated in metastatic breast cancer. PTPRE was found to play an important role in HER2-mediated transformation as an activator of SRC (Gil-Henn and Elson 2003). SHP2 also has an oncogenic function in ERK activation in HER2-positive breast cancer (Bentires-Alj et al. 2006). However, the function of some PTPs may vary according to the context and stage of breast cancer development. For example, PTPN12 has been identified as a tumor suppressor that is frequently altered in TNBC, with effects on multiple tyrosine phosphorylation-dependent signaling pathways (Sun et al. 2011). In contrast, PTPN12 has also been implicated in regulating levels of reactive oxygen species, thereby promoting resistance to oxidative stress and favoring tumorigenesis. It was reported that deletion of PTPN12 in human and mouse breast tumor cells decreased tumorigenic and metastatic potential in vivo, consistent with PTPN12 itself being a therapeutic target in certain contexts (Harris et al. 2014).

In this study, we focused on PTPN23, which is a ubiquitously expressed classical PTP composed of five domains (Gingras et al. 2009a; Bissig and Gruenberg 2014). The BRO1-like and V domains are important in ESCRT (endosomal sorting complexes required for transport) sorting of several cell surface receptors, such as EGFR and integrin (Ali et al. 2013; Kharitidi et al. 2015). The gene encoding PTPN23 resides on chromosome $3 \mathrm{p} 21$, a region that is frequently homozygously or hemizygously deleted in various human tumors, also consistent with a tumorsuppressive function (Senchenko et al. 2004; Angeloni 2007). The observation that the catalytic activity of PTP-TD14 (the rat PTPN23 ortholog) is required to suppress RAS-mediated transformation of fibroblasts in soft agar assay (Cao et al. 1998) emphasized the importance of the C-terminal catalytic domain in its tumor suppressor function. In addition, suppression of PTPN23 has also been implicated in miR-142-3p-mediated increases in soft agar colony formation by a human testicular germ cell tumor cell line, whereas overexpression of PTPN23 impedes xenograft growth of testicular germ cell tumors in vivo (Tanaka et al. 2013). Furthermore, in a transgenic mouse study, hemizygous deletion of PTPN23 results in sporadic lung adenoma and B-cell lymphoma and promotes the establishment and progression of Myc-driven lymphoma (Manteghi et al. 2016). In the same study, it was reported that loss of PTPN23 in patients coincided with poor survival (Manteghi et al. 2016).

Our laboratory performed RNAi-mediated loss-of-function screens to test the effects of suppressing systematically the expression of PTP family members in threedimensional cell culture models. We identified several PTPs as novel negative and positive regulators of HER2 signaling in the control of cell polarity, migration, and invasion (Lin et al. 2011; Ramesh et al. 2015). Interestingly, only PTPN23 was shown to inhibit mammary epithelial cell motility and invasion in a HER2-independent manner by impairing the integrity of adheren junctions through regulating the phosphorylation status of E-cadherin and $\beta$-catenin (Lin et al. 2011). However, the extent to which PTPN23 is tumor-suppressive in breast oncogenesis and the mechanism by which it may exert such a function are still unknown.

Here we report a loss-of-function study in which PTPN23 was suppressed in transplantation and xenograft mouse models to investigate a potential tumor-suppressive role in breast tumorigenesis. We established a mammary gland orthotopic transplantation model with PTPN23-deficient Comma 1D $\beta$ (CD) cells and observed the incidence of basal-like breast tumors in PTPN23-deficient animals. Importantly, we observed that PTPN23 recognized FYN as a physiological substrate, functioning as a negative regulator of FYN activity in vitro and in vivo. We demonstrated that tumorigenesis induced by loss of PTPN23 in xenograft models was abated either 
by treatment with AZD0530, an SRC family kinase (SFK)specific inhibitor, or by cosuppression of PTPN23 and FYN by CRISPR-CAS9 gene editing. Overall, our study links the tumor-suppressive function of PTPN23 in breast tumorigenesis to regulation of FYN phosphorylation and activity, suggesting that FYN may be a therapeutic target for breast cancer defined by hemizygous or homozygous loss of PTPN23.

\section{Results}

Expression of PTPN23 was down-regulated and positively correlated with overall survival rate in breast cancer patients

We analyzed data from 1069 breast cancer patients in TCGA (The Cancer Genome Atlas) to investigate whether there was a relationship between PTPN23 expression and overall survival rate. The data indicated that expression of PTPN23 was positively correlated with overall survival in breast cancer patients (Fig. 1A). In contrast, expression of PTPRG, the only other classical PTP gene also localized in chromosome 3 (Andersen et al. 2004), displayed no significant correlation with overall survival ratio in breast cancer patients (Fig. 1B). To examine further the pathological significance of PTPN23 expression in breast cancer occurrence, we performed immunohistochemistry (IHC) staining with antibodies against PTPN23. Two breast cancer tissue microarrays (TMAs), including normal breast cores as controls (TMA US Biomax, Inc.), were stained and scored for positive staining (Fig. 1C). Consistent with clinical correlation, we observed in the first tissue array a significant down-regulation of PTPN23 expression in breast cancers $(n=100)$ compared with normal breast core tissues $(n=10, P<0.0001)$ (Fig. 1D), regardless of their tumor stages (stage I $[n=5]$, stage II [ $n=74]$, and stage III $[n=21]$ ) (Fig. 1E). More importantly, TNBC patients in the second tissue array had the lowest PTPN23 expression among all receptor status subgroups $\left(\mathrm{ER}^{+}[n=4], \mathrm{PR}^{+}\right.$ $[n=3]$, HER $^{+}[n=15]$, and TNBC $\left.[n=33] ; P=0.0017\right)$, suggesting that deletion or suppression of PTPN23 was a potential risk factor for TNBC occurrence and progression (Fig. 1F). Overall, clinical analyses from TCGA database and TMA revealed that attenuation of PTPN23 expression in breast cancer, especially TNBC, was correlated with poor long-term survival rate.

\section{Suppression of PTPN23 in CD cells promoted tumor formation in a $B A L B / c$ orthotopic transplantation model}

Since the homozygous deletion of PTPN23 results in embryonic lethality in a transgenic mouse model (Gingras et al. 2009a), we took advantage of an orthotopic mammary gland transplantation model using CD cells to investigate the tumor-suppressive function of PTPN23 in breast tumorigenesis. CD cells, a mammary gland epithelial progenitor cell line derived from mammary gland of midphase pregnant $\mathrm{BALB} / \mathrm{c}$ mice, are able to differentiate into cells with both luminal and basal epithelial cell properties. As a consequence of their immortal nature and pluripotency, CD cells are used widely in studies of mammary gland development and breast cancer tumorigenesis (Danielson et al. 1984; Ibarra et al. 2007). The experimental scheme is summarized in Figure 2A. First, we selected two effective shRNAs (shPtpn23 \#2913 and \#3971) to suppress PTPN23 in CD cells (Fig. 2B) and then injected these cells into cleared abdominal fat pads (fourth pair) of 3-wk-old female BALB/c mice (12 mice per group). Although Ptpn23 knockdown did not alter $\mathrm{CD}$ cell proliferation in a CellTiter-Glo viability assay (Supplemental Fig. S1A), we did observe the onset of breast tumors in Ptpn23 knockdown cohorts from week 20 post-transplantation. In the tumor-free curve (Fig. 2C), seven of 12 mice in the shPtpn23 \#2713 cohort and eight of 12 in the shPtpn23 \#3971 cohort developed palpable breast tumors in one or both transplanted fat pads within 56 wk, whereas the shLuc (shRNA against firefly luciferase) cohort remained tumor-free (as assessed by palpation). All breast tumors grew at a high rate in vivo, and mice were euthanized above a tumor burden of $d \geq 20 \mathrm{~mm}$. In fact, six of 12 littermates in the shPtpn23 \#2713 cohort and seven of 12 in the shPtpn23 \#3971 cohort (Fig. 2D) were euthanized due to aggressive tumor progression by the end of $56 \mathrm{wk}$.

Histologically, we categorized CD cell-derived breast tumors from 10 mice in the shPtpn23 \#2713 cohort and nine in the shPtpn23 \#3971 cohort into two major subtypes (Supplemental Fig. S1B). One was adenosquamous carcinoma and the other was anaplastic carcinoma (Fig. $2 \mathrm{~F}$ ), both of which are basal-like breast tumor subtypes. Additionally, we observed lung macrometastasis in one littermate from each shPtpn23 cohort. The shRNA cassettes coexpress GFP; using fluorescence microscopy, we detected GFP in shPtpn23 tumor sections but not in normal mammary glands (Supplemental Fig. S1C), confirming that the tumors were derived from PTPN23deficient CD cells. In addition, we demonstrated that PTPN23 was efficiently suppressed in shPtpn23 CD cells during tumor development in vivo using IHC with fluorescence detection (Supplemental Fig. S1D). Approximately $40 \%$ of cells were PTPN23-positive in normal (44\%) and control shLuc $(42 \%)$ mammary glands, whereas only $12 \%$ were positive in shPtpn23 tumors (Supplemental Fig. S1E). Expression of PTPN23 in shPtpn23 tumor lysates was as low as that observed in shPtpn23 CD cells, suggesting sustained knockdown of Ptpn23 during tumorigenesis (Supplemental Fig. S1F). In conclusion, this is consistent with a tumor suppressor function of PTPN23, with suppression of PTPN23 promoting CD cells to develop into basal-like breast tumors in orthotopically transplanted $\mathrm{BALB} / \mathrm{c}$ mice.

Loss of PTPN23 sustained FYN activity, with concomitant phosphorylation of $\beta$-catenin on Tyr142 in $C D$ cells

Our previous study indicated that depletion of PTPN23 elevated the activity of SFKs as well as tyrosine phosphorylation of $\beta$-catenin on Tyr142 (Lin et al. 2011). Furthermore, in a loss-of-function screen of classical PTPs, 
A

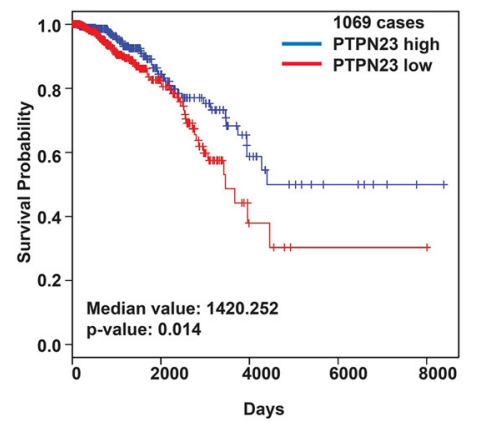

B

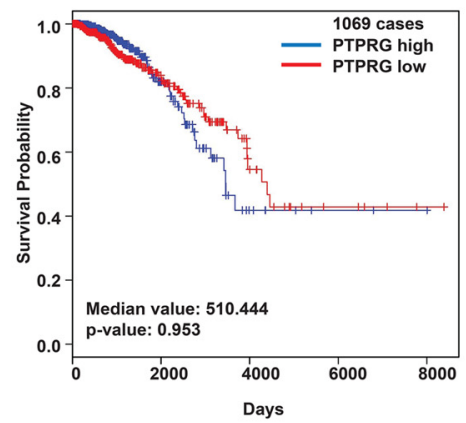

C

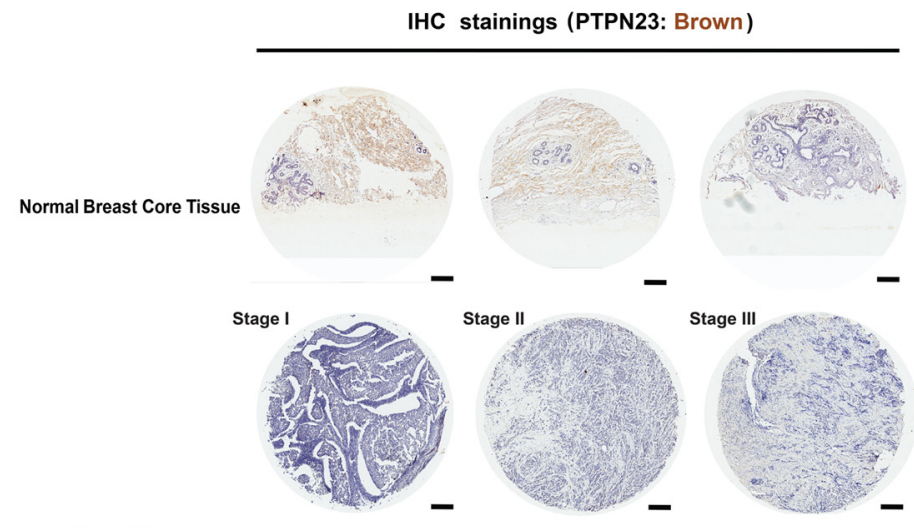

Breast Tumor
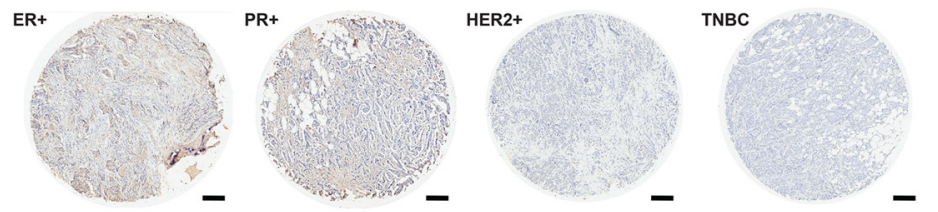

D

E

$\mathbf{F}$
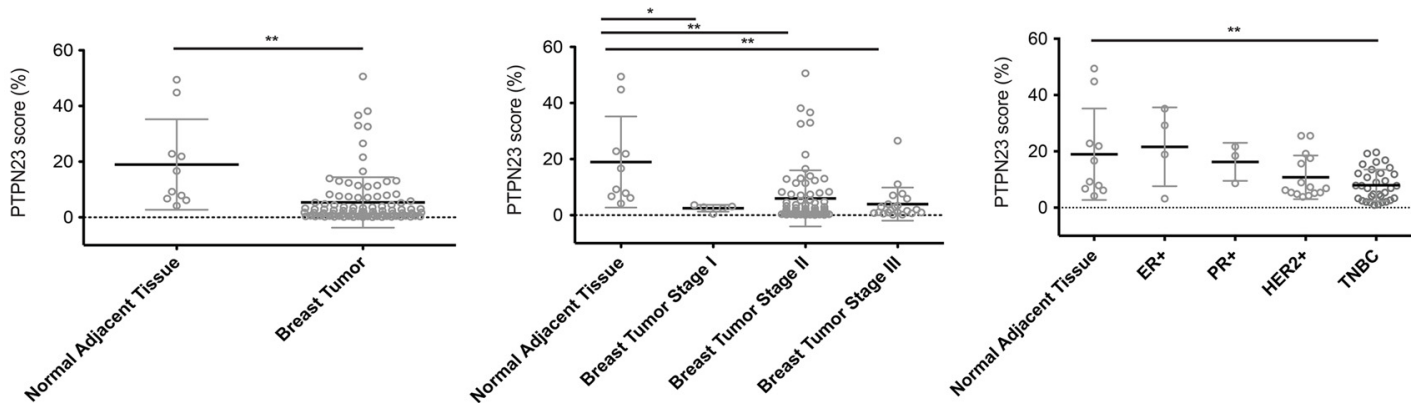

Figure 1. Expression of PTPN23 was down-regulated and correlated positively with overall survival rate in breast cancer patients. $(A, B) \mathrm{A}$ data set of 1069 breast cancer cases was extracted from TCGA database. PTPN23 and PTPRG expression was stratified as high versus low against median expression, and overall survival probability between these two subgroups was plotted. $(A)$ PTPN23. $(B)$ PTPRG. The $P$-value was calculated with two-tailed log-rank (Mantel-Cox) test. $(C)$ Representative IHC staining of PTPN23 (brown) in normal adjacent tissue (top panel) and breast tumor specimens classified by the TNM staging system (middle panel) as well as receptor status (bottom panel). Bar, $200 \mu \mathrm{m}$. $(D)$ Expression of PTPN23 in normal adjacent tissue $(n=10)$ and breast tumor specimens $(n=100)$ was scored by Aperio ImageScope software and plotted as mean + SD. (E) Expression of PTPN23 in individual TNM tumor stage I $(n=5)$, stage II $(n=74)$, and stage III $(n=21)$ subgroups was also plotted as mean \pm SD, with that of normal breast core tissues $(n=10)$ as control. $(F)$ Expression of PTPN23 in $\mathrm{ER}^{+}(n=4), \mathrm{PR}^{+}(n=3), \mathrm{HER}^{+}(n=15)$, and TNBC $(n=33)$ was plotted as mean $\pm \mathrm{SD}$, with that of normal breast core tissues $(n=10)$ as control. $\left(^{*}\right) P<0.05$, unpaired two-tailed Student's $t$-test; $\left(^{* *}\right) P<0.01$, unpaired two-tailed Student's $t$-test. 
A

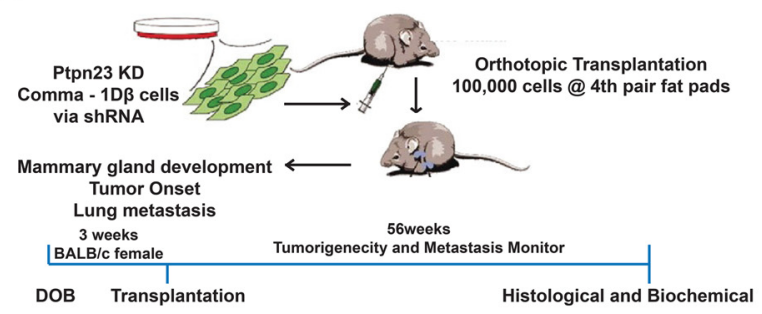

C

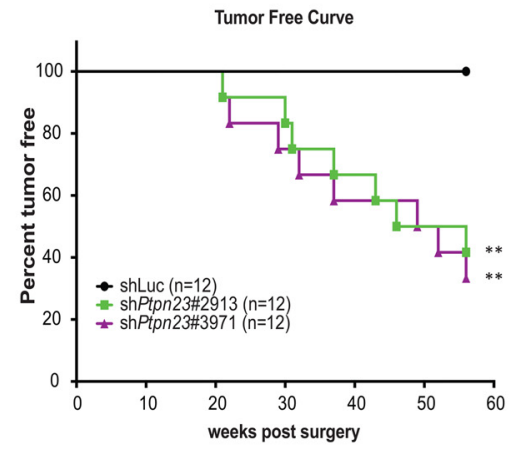

B

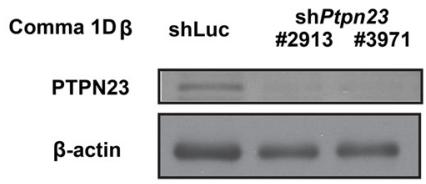

D

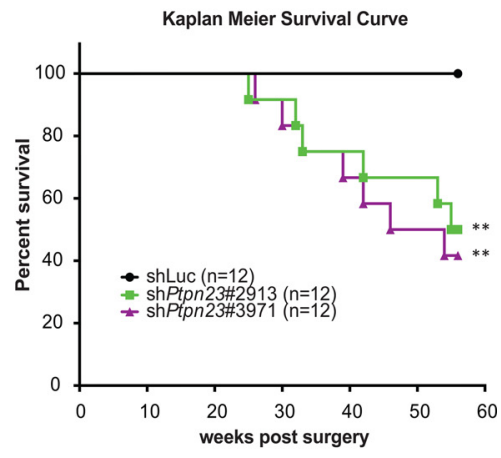

E

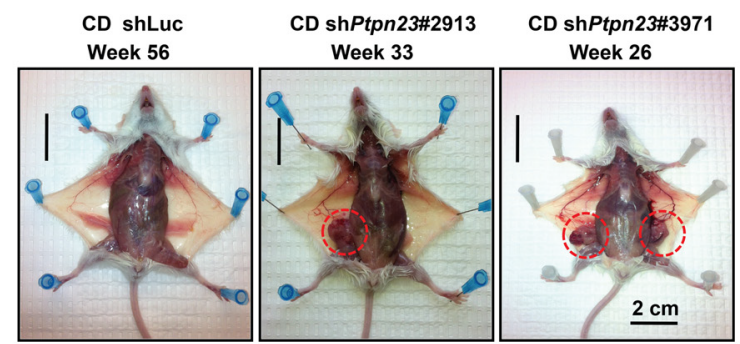

$\mathbf{F}$
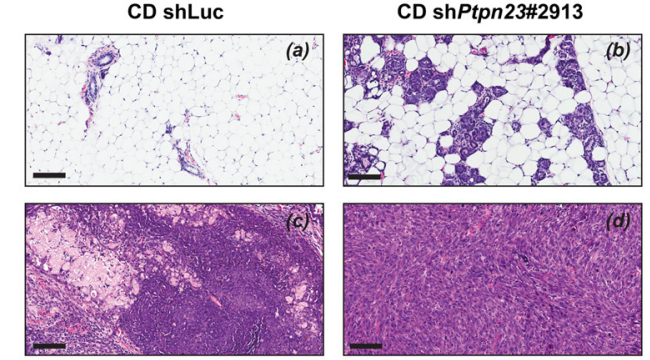

CD shPtpn23\#2913

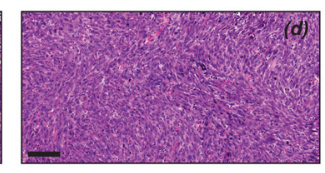

CD shPtpn23\#3971

Figure 2. Suppression of PTPN23 in CD cells promoted breast tumor formation in a BALB/c mouse orthotopic transplantation model. $(A)$ Schematic summary of the use of CD cells in a mammary gland orthotopic transplantation model in BALB/c mice. $(B)$ Total cells lysates from Ptpn23 knockdown (shPtpn23 \#2913 and \#3971) and control (shLuc) CD cell lines were immunoblotted for PTPN23 and $\beta$-actin. (C) All BALB/c mice were monitored for any palpable breast tumor two times a week for $56 \mathrm{wk}$. The percentage of tumor-free animals in three

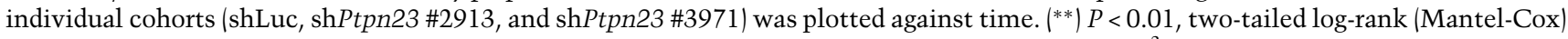
test. $(D)$ Mouse mortality, either direct deceased or by euthanasia with a heavy tumor burden $\left(>4 \mathrm{~cm}^{3}\right)$, was documented in Kaplan-Meier survival curves for all three cohorts (shLuc, shPtpn23 \#2913, and shPtpn23 \#3971). ${ }^{* *}$ ) $P<0.01$, two-tailed log-rank (Mantel-Cox) test. (E) Representative animal necropsy images from each cohort, showing mammary glands in the CD shLuc cohort and breast tumors (red circle) in the CD shPtpn23 \#2913 and hPtpn23 \#3971 cohorts. Bar, $2 \mathrm{~cm} .(F)$ Representative H\&E staining images for repopulated mammary glands and two major breast tumor subtypes derived from CD cells in BALB/c mice. (Panel $a$ ) Mammary gland reconstituted by CD shLuc cells. (Panel b) Atypical hyperplasia and high-grade MIN (mammary intraepithelial neoplasis) developed from CD shPtpn23 \#2913 cells. (Panel c) Adenosquamous carcinoma developed from CD shPtpn23 \#2913 cells. (Panel d) Anaplastic carcinoma with traces of stroma developed from CD shPtpn23 \#3971 cells. Bar, $100 \mu \mathrm{m}$. 
A

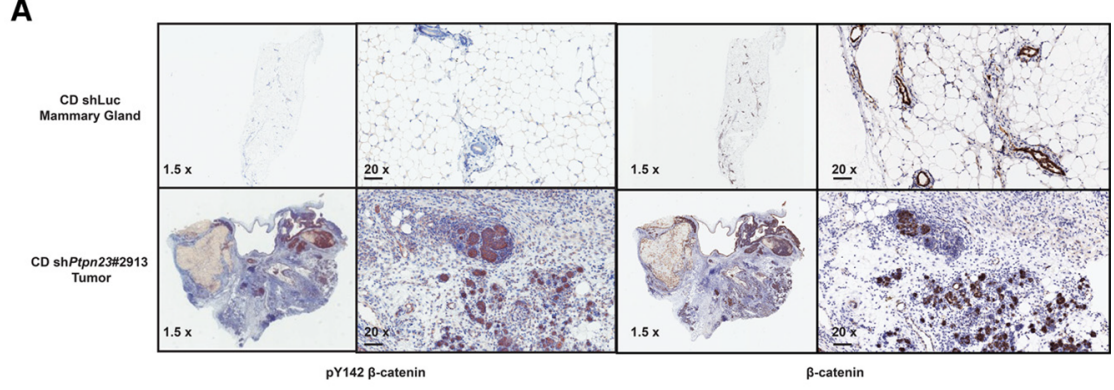

B

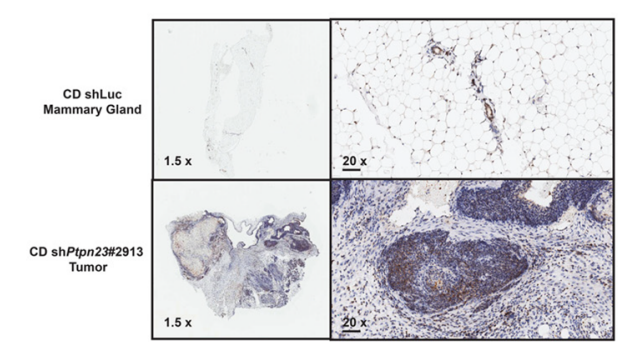

C

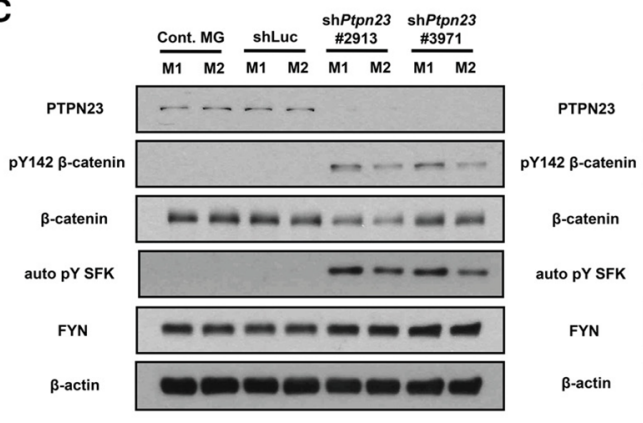

D

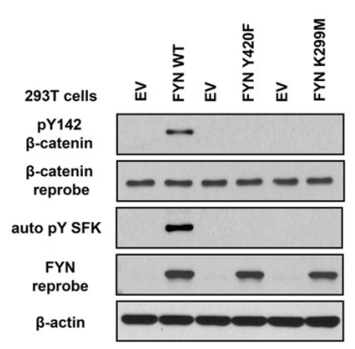

E

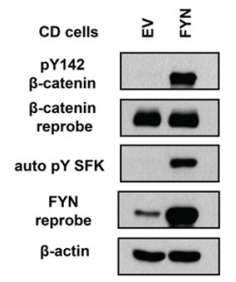

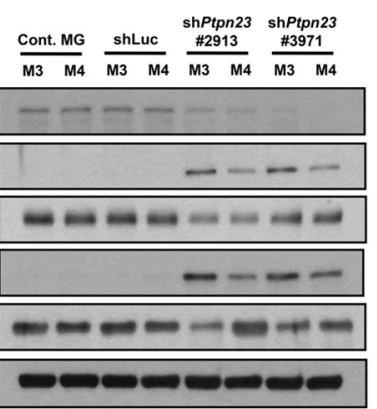

F

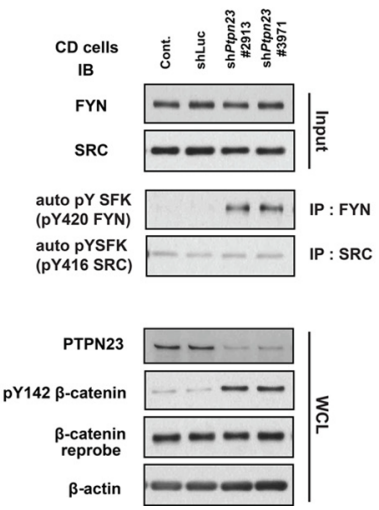

Figure 3. Loss of PTPN23 sustained FYN activity, with concomitant phosphorylation of $\beta$-catenin on Tyr142 in CD cells. (A) IHC staining of pTyr142 $\beta$-catenin (left) and $\beta$-catenin (right) of a mammary gland section following CD shLuc cell transplantation (top panel) and a breast tumor section following CD shPtpn23 \#2913 cell transplantation (bottom panel). Nuclei were also stained (blue). Bar in 20-fold magnification images, $50 \mu \mathrm{m}$. (B) IHC staining of the autophosphorylation site pTyr of SFK (active) from a mammary gland section with CD shLuc cell transplantation (top panel) and a breast tumor section with CD shPtpn23 \#2913 cell transplantation (bottom panel). Nuclei were also stained (blue). Bar in 20-fold magnification images, $50 \mu \mathrm{m} .(C)$ Mammary glands from both untransplanted and CD shLuc cohorts as well as tumor tissues from both shPtpn23 \#2913 and shPtpn23 \#3971 cohorts were frozen and lysed. Four replicates of tissue lysates (M1 to M4) from each group (untransplanted normal mammary glands [Cont. MG], shLuc, shPtpn23 \#2913, and shPtpn23 \#3971) were immunoblotted with antibodies against PTPN23 to confirm the knockdown efficiency. Tissue lysates were also immunoblotted against pTyr142 and total $\beta$-catenin and pTyr-SFK and total FYN. HEK293T cells were transiently transfected with empty vector $(\mathrm{EV})(D, E)$ and vectors that express wide-type or Y420F or K299M mutant FYN $(D)$. $(E)$ A CD cell line stably expressing ectopic FYN was also established. All cell lysates were harvested to probe for pTyr142 and total $\beta$-catenin and pTyr-SFK (pY420 FYN) and total FYN, with $\beta$-actin as a loading control. $(F)$ Parental, shLuc, shPtpnN23 \#2913, and shPtpn23 \#3971 CD cell lines were stimulated with complete growth medium for 30 min after overnight serum starvation. PTPN23, pTyr142, and total $\beta$-catenin were probed by immunoblotting. FYN and SRC were immunoprecipitated followed by immunoblotting with pTyr-SFK antibody to check the activation status of FYN and SRC in all four CD cells. depletion of which led to elevated Tyr142 phosphorylation in $\beta$-catenin, we also identified PTPN23 as one of the top hits (Supplemental Fig. S2A,B). In order to investigate the underlying mechanism by which suppression of PTPN23 in CD cells led to breast oncogenesis in the transplantation model, we tested first whether the same regulation would occur in vivo. Therefore, we performed IHC staining of tissue samples with specific antibodies to demonstrate the phosphorylation status of $\beta$-catenin on Tyr142 (Fig. 3A) and the Tyr autophosporylation site in the activation loop of SFKs (Fig. 3B). The percentage of positive staining of pTyr142 (Supplemental Fig. S2C) and total $\beta$-catenin (data not shown) was scored and calculated for each sample. As expected, we detected a higher percentage of cells that stained positive for pTyr142 $\beta$-catenin with strong intensity in shPtpn23 tumors $(66 \%)$ compared with shLuc mammary glands $(6 \%)$ or normal mammary glands $(5 \%)$. Consistently, the ratio of pTyr 142 over total $\beta$-catenin staining in shPtpn23 tumors $(66 \%)$ was significantly higher than in shLuc $(14 \%)$ and normal (15\%) mammary glands (Supplemental Fig. S2D). Consistent with the elevated $\beta$-catenin phosphorylation on Tyr142, we observed that a higher percentage of cells stained positive for the pTyr autophosphorylation site in 
SFKs in shPtpn23 tumors $(51 \%)$ than in shLuc $(18 \%)$ or normal (13\%) mammary glands (Supplemental Fig. S2E). To confirm the result, tissue lysates from mammary glands (shLuc), breast tumors (shPtpn23 \#2913 and \#3971), and untransplanted normal mammary glands were collected and subjected to immunoblotting. As expected, we detected increased $\beta$-catenin and SFK phosphorylation in tumor lysates compared with mammary gland controls (Fig. 3C).

As functional kinases, activated SFKs are able to convey signals through substrate phosphorylation; however, the question remained which SFK was important in this context? The Dunach laboratory (Piedra et al. 2003) reported that increased phosphorylation of Tyr142 in $\beta$-catenin in epithelial cells was accompanied by the activation of FYN kinase. Furthermore, FYN was shown to phosphorylate $\beta$-catenin on Tyr142 in vitro (Piedra et al. 2003). Therefore, we investigated whether Tyr142 of $\beta$-catenin could also be phosphorylated by FYN in CD cells. We confirmed the expression of FYN in CD cells (Supplemental Fig. S2F) and demonstrated that only wild-type, but not Y420F (kinase-inactivated) or K299M (kinase-dead) mutants of, FYN could phosphorylate $\beta$-catenin on Tyr142 in $293 \mathrm{~T}$ cells (Fig. 3D). Finally, we detected the robust phosphorylation of $\beta$-catenin on Tyr142 in CD cells in which FYN was stably expressed (Fig. 3E). These data indicate that Tyr142 of $\beta$-catenin is a substrate of FYN.

To provide further evidence that the activation of FYN was enhanced in CD cells following suppression of PTPN23, we serum-starved shLuc, shPtpn23 \#2913, shPtpn23 \#3971, and parental CD cells overnight and restimulated them in complete growth medium for $30 \mathrm{~min}$. FYN and SRC were immunoprecipitated from the cell lysates, and activation of these two kinases was examined. Compared with parental and shLuc CD cells, we observed increased autophosphorylation of FYN Tyr420 in both shPtpn23 \#2913 and shPtpn23 \#3971 CD cells. However, autophosphorylation of Tyr416 in SRC remained unchanged among these samples (Fig. 3F). Interestingly, the phosphorylation status of $\beta$-catenin on Tyr142 was also dramatically elevated upon PTPN23 suppression (Fig. 3F). Taken together, these data indicate both in vivo and in vitro that suppression of PTPN23 in CD cells enhanced FYN autophosphorylation and activation, with a concomitant increase in phosphorylation of $\beta$-catenin on Tyr142.

\section{PTPN23 dephosphorylated FYN on Tyr420}

In order to validate FYN as a bona fide substrate of PTPN23, we first performed in vitro substrate trapping experiments in CD cells. This approach, which has served as an important tool to identify putative PTP substrates, is based on stabilization of a PTP-substrate complex following mutation of an invariant Asp residue in the WPD loop to Ala (WPE loop in PTPN23) or mutation of the nucleophilic Cys to Ser in the PTP signature motif (Flint et al. 1997). We introduced both Flag-tagged C1392S and E1357A mutants of PTPN23 in CD cells to compare their interaction with FYN with that of wild-type PTPN23.
Analysis by coimmunoprecipitation illustrated greater complex formation between these PTPN23 mutants and FYN compared with the wild-type phosphatase (Fig. 4A). We repeated the substrate-trapping experiment in CD cells and assessed the phosphorylation status of FYN with antibodies against either the autophosphorylation site (pTyr420 FYN) or the inhibitory C-terminal pTyr phosphorylation site (pTyr531 FYN). In agreement with what we observed in tissue samples (Fig. 3B), the portion of FYN that coimmunoprecipitated with the PTPN23 CS mutant was highly phosphorylated on Tyr420 but not on Tyr531 (Fig. 4B). Importantly, Y420F, but not the Y531F mutant of FYN, did not autophosphorylate or associate with the PTPN23 CS mutant (Fig. 4C). To examine substrate selectivity, we repeated these assays using PTPN23 CS together with two other SFK members, SRC and YES, in CD cells. Unlike FYN, we detected no association between the PTPN23 CS mutant and these two kinases even with their activating tyrosine residue phosphorylated (Supplemental Fig. S3A-C).

To consolidate the substrate-trapping data, we investigated whether FYN was a direct substrate of PTPN23 in a dephosphorylation assay in vitro. We enriched Y531F FYN as substrate by immunoprecipitation following transient expression in CD cells. Following incubation of the wild-type PTPN23 catalytic domain with Y531F FYN, the extent of phosphorylation of pTyr420 in FYN was diminished, whereas incubation with C1392S mutant PTPN23 did not result in dephosphorylation (Fig. 4D). Sequence alignments of all classical PTPs revealed that PTPN23 harbors three amino acid residue alterations in its catalytic motif (His1223, Glu1357, and Ser1394) compared with the normal consensus. In order to test whether any of the three alterations had an impact on enzymatic activity, we purified the PTPN23 catalytic domain with site-directed mutations at each of the three residues (H1223Y, E1357D, and S1394A) as well as a triple-mutant form (H1223Y) E1357D/S1394A) to convert to the consensus sequence. Although the three single-mutant forms of the PTPN23 catalytic domain (H1223Y, E1357D, and S1394A) dephosphorylated pTyr 420 of FYN comparably with the wildtype PTPN23 catalytic domain, the activity of the triple mutant (H1223Y/E1357D/S1394A) was elevated (Fig. 4D). In summary, the data revealed FYN as a bona fide substrate of PTPN23, and its activity may be inhibited by PTPN23 through direct dephosphorylation of Tyr420 in the activation loop.

Treatment of AZD0530, a specific SFK inhibitor, offsets BT474 breast tumor progression induced by PTPN23 suppression

In parallel with the transplantation model, we also investigated the function of PTPN23 on tumor progression in a SCID/Beige xenograft model with BT474 cells, a HER2 ${ }^{+}$ breast cancer cell line. The BT474 cells were engineered to express a luciferase cassette to permit examination of tumor development via living bioluminescence imaging. Consistent with the results of our transplantation model, suppression of PTPN23 in BT474 cells via two individual 
A

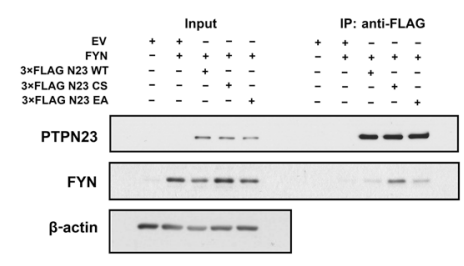

B

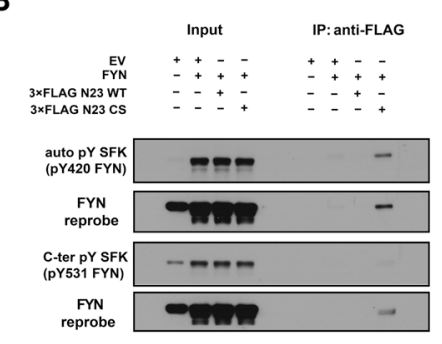

C

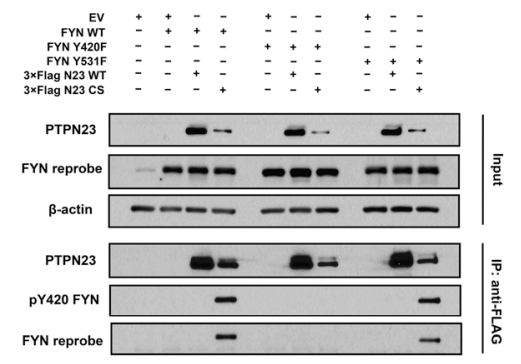

D

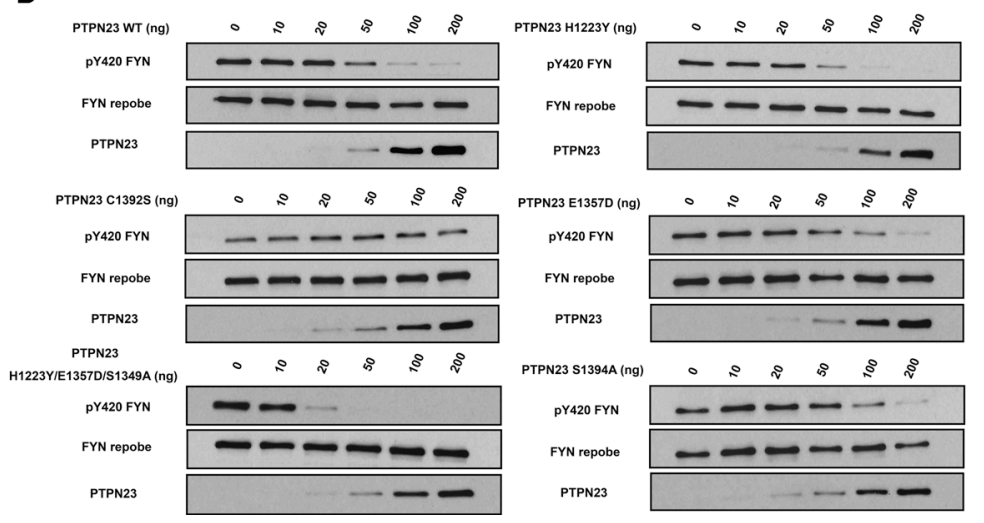

Figure 4. PTPN23 dephosphorylated FYN on Tyr420. (A) CD cells were transiently transfected with mammalian expression vectors for both FYN and Flag-tagged wild-type (3xFlag N23 wild-type) or substrate-trapping mutant (3xFlag N23 C1392S and E1357A) forms of PTPN23. Cell lysates were harvested and immunoprecipitated with Flag antibody-conjugated magnetic beads. The interactions of FYN with wild-type, C1392S, or E1357A PTPN23 were assessed by immunoblotting with the appropriate antibodies. $\beta$-Actin was probed as the loading control. $(B)$ $\mathrm{CD}$ cells were transiently transfected with the indicated vectors, lysed, and immunoprecipitated with Flag antibody-conjugated magnetic beads. The immunoprecipitates and $10 \%$ input lysates were probed with the indicated antibodies. $(C)$ Potential PTPN23 dephosphorylation sites were investigated using kinase-impaired (Y420F) and constitutively active (Y531F) mutant forms of FYN. CD cells transiently transfected with vectors expressing wild-type, Y420F, and Y531F FYN in combination with PTPN23 wild-type and C1392S vectors, as indicated, were lysed and immunoprecipitated with Flag antibody-conjugated magnetic beads. The immunoprecipitates and $10 \%$ input cell lysates were probed with the indicated antibodies. $\beta$-Actin was probed as the loading control. $(D)$ Y531F FYN was expressed in CD cells and immunoprecipitated with FYN antibody. Equal amounts of the immunoprecipitate were incubated with a panel of purified PTPN23 catalytic domains at the indicated amounts for $30 \mathrm{~min}$ at $30^{\circ} \mathrm{C}$, including the wild-type and five mutant forms of PTPN23 (C1392S, H1223Y, E1357D, S1394A PTPN23, and C1392S/H1223Y/E1357D PTPN23). The reaction was terminated by addition of sample buffer, and dephosphorylation of pTyr420 in FYN was assessed by immunoblotting with the indicated antibodies.
shRNAs accelerated growth of breast tumors, as measured by size and net weight, in this xenograft model (Supplemental Fig. S4A-E), consistent with a general tumorsuppressive role for PTPN23 in different breast cancer subtypes.

Considering that expression of PTPN23 was downregulated in a broad array of breast cancers (Fig. 1) and that PTPN23 negatively modulated FYN kinase activity by dephosphorylation of Tyr420 in vitro (Fig. 4), we tested whether this mechanism also explained the tumor-suppressive role of PTPN23 in breast cancer models. We selected an SFK-specific inhibitor, AZD0530 (Saracatinib), to inhibit the kinase activity of FYN in vivo (Hennequin et al. 2006). If PTPN23 exerted its tumor suppressor function through the regulation of FYN activity in vivo, we anticipated that treatment with AZD0530 may alleviate breast tumor progression in the xenograft model. As shown in Figure 5A, tyrosine phosphorylation of SFK in the activation loop was elevated in PTPN23-deficient BT474 cells, and this phosphorylation was abrogated upon treatment with $5 \mu \mathrm{M}$ AZD0530, confirming the inhibitory effect of this compound. We injected shPTPN23
\#1058 and shCont BT474 cells bilaterally into the left and right abdominal mammary gland fat pads (Fig. 5C, ventral view) of 8-wk-old female SCID/Beige mice. Two weeks after injection, $25 \mathrm{mg} / \mathrm{kg}$ AZD0530 was administered orally every $3 \mathrm{~d}$ for $6 \mathrm{wk}$ to one cohort of animals $(n=8)$, whereas vehicle control (5\% DMSO diluted in saline) was administered to the other cohort $(n=9)$ (Fig. 5B). We monitored body weight and calibrated tumor size of each animal every $3 \mathrm{~d}$ for $6 \mathrm{wk}$. There was no detectable gain or loss in body weight between the two cohorts (Fig. 5C); however, compared with vehicle, AZD0530 treatment did significantly delay the tumor progression of PTPN23-deficient BT474 cells (54-d treatment: $270 \mathrm{~mm}^{3}$ vs. $100 \mathrm{~mm}^{3}$ ) (Fig. 5D). After euthanasia, all of the tumors were dissected and measured ex vivo. Compared with the shPTPN23 vehicle cohort, both tumor volume and weight from the shPTPN23-AZD0530 cohort were reduced to levels observed in the shCont vehicle cohort (Fig. 5E,F). Furthermore, representative bioluminescence images of one littermate from each treatment group on day 14 and day 46 illustrated the delayed tumor growth from the shPTPN23 AZD0530 
A

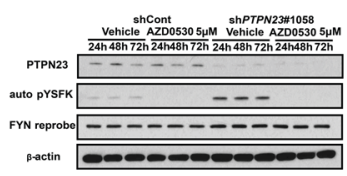

C

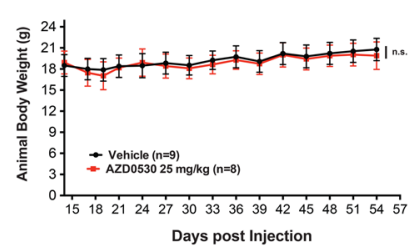

E

G
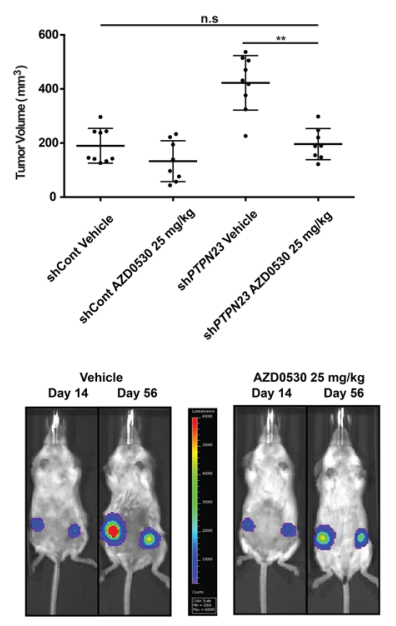

I

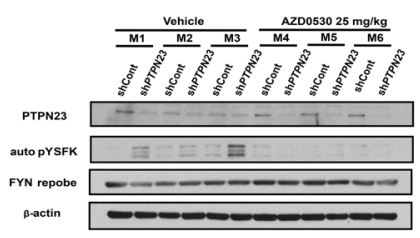

B

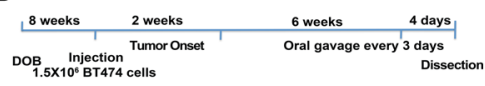

D

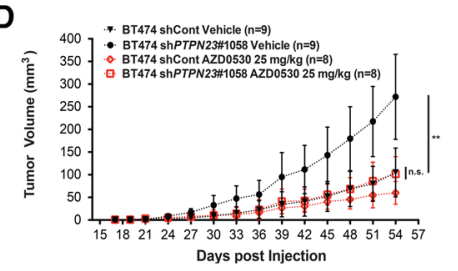

$\mathbf{F}$

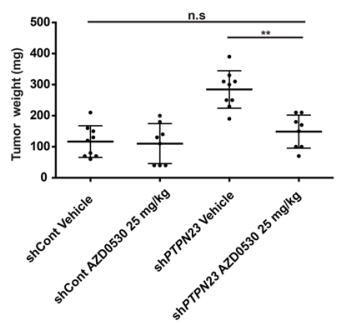

H
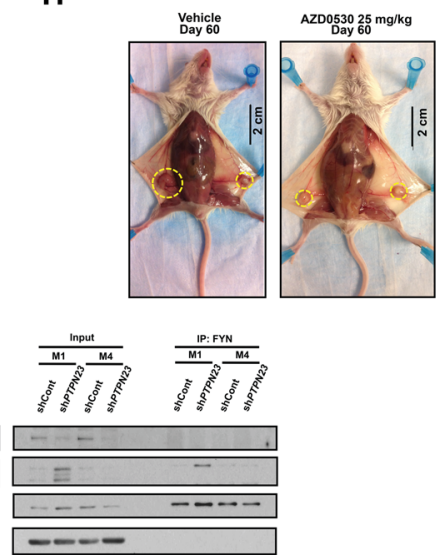

Figure 5. Treatment with AZD0530, a specific SFK inhibitor, attenuated BT474 breast tumor progression induced by PTPN23 suppression. (A) Prior to the xenograft experiment, inhibition of kinase activity by AZD0530 as well as PTPN23 knockdown efficiency were examined by immunoblotting with the indicated antibodies in BT474 cell lines (shCont and shPTPN23 \#1058). $\beta$-Actin was probed as the loading control. (B) Schematic plan for the mammary gland orthotopic xenograft model in SCID/Beige mice using BT474 cells and treatment with AZD0530 (saracatibnib). (C) shPTPN23 \#1058 and shCont BT474 cells $\left(1.5 \times 10^{6}\right.$ cells $)$ were orthotopically injected into the left and right abdominal mammary glands (ventral view), respectively. Each animal was administrated either vehicle or $25 \mathrm{mg} / \mathrm{kg}$ AZD0530. Body weight was monitored in grams and plotted every $3 \mathrm{~d}$ after 14-d tumor onset. (n.s.) Not significant, two-tailed log-rank (Mantel-Cox) test. $(D)$ For each xenograft tumor, the length and width were measured with calipers (in millimeters) every $3 \mathrm{~d}$ before treatment. The tumor volume was calculated in cubic millimeters [volume $=($ width $\times$ width $\times$ length $) / 2]$, and the mean \pm SD was plotted for the vehicle-treated and $25 \mathrm{mg} /$ kg AZD0530-treated cohorts. (n.s.) Not significant; $\left({ }^{* *}\right) P<0.01$, two-tailed log-rank (Mantel-Cox) test. $(E, F)$ Each tumor in the four individual treatment subgroups was collected after euthanasia, with its length and width calibrated in millimeters and its volume calculated in cubic millimeters [volume $=\mid$ width $\times$ width $\times$ length $/ 2$ ]. $(E)$ The mean \pm SD of tumor volume for the four indicated subgroups was plotted. Furthermore, the net weight of each tumor was measured in milligrams. $(F)$ The mean $\pm \mathrm{SD}$ of tumor weight for the four indicated subgroups was plotted. (n.s.) Not significant; $\left.{ }^{* *}\right) P<0.01$, two-tailed MannWhitney test. $(G)$ Bioluminescence in vivo imaging was applied to show the effect of AZD0530 treatment. Representative images were taken at the first and last day of the treatment. (Right) BT474 shCont cells. (Left) BT474 shPTPN23 \#1058 cells. Bioluminescence signals were quantitated by Living Image software. The intensity is represented by rainbow scale, ranging from 2000 to 60,000 counts. $(H)$ Representative animal images showing the effect of AZD0530 treatment. (Right) BT474 shCont cells. (Left) BT474 shPTPN23 \#1058 cells. Bar, $2 \mathrm{~cm}$. (I) Dissected tumor tissues from both vehicle-treated (M1, M2, and M3) and AZD0530-treated (M4, M5, and M6) cohorts were frozen, lysed, and immunoblotted with the indicated antibodies to confirm the efficiency of PTPN23 knockdown and AZD0530 inhibition. All tumor tissue lysates were also immunoprecipitated with FYN antibody followed by immunoblotting with pTyr-SFK (pY420 FYN) antibody and reprobing of FYN (M1 and M4 are shown). $\beta$-Actin was probed as the loading control.

cohort (Fig. 5G). The dissection image of the same pair of mice on day 60 also contrasted the actual tumor size, suggesting that SFK inhibition offset the effect of PTPN23 suppression on tumor progression in BT474 xenografts (Fig. 5H).

Finally, we harvested tumor samples from all littermates in both treatment groups for biochemical examination. First, we randomly paired tumor tissues from three mice treated with vehicle (M1, M2, and M3) and three mice treated with AZD0530 (M4, M5, and M6), immunoprecipitated FYN, and then compared the extent of phosphorylation of Tyr420 by immunoblotting. In the vehicle treatment group, shPTPN23 tumors showed a higher phosphorylation status of Tyr420 in FYN than in the shCont cohort; however, FYN Tyr420 autophosphoryla- tion was abrogated in both shCont and shPTPN23 tumor lysates following AZD0530 treatment (Fig. 5I; Supplemental Fig. S5A,B). Second, we performed IHC staining for H\&E (Supplemental Fig. S5C, left), PTPN23 Supplemental Fig. S5C, (middle), and SFK autophosphorylation (Supplemental Fig. S5C, right) on all tumor slides and scored for positive staining (Supplemental Fig. S5D,E). Suppression of PTPN23 enhanced tyrosine phosphorylation of SFK; however, this increase was dramatically inhibited following AZD0530 administration (Supplemental Fig. S5C-E). In conclusion, the accelerated tumor progression in the PTPN23-deficient BT474 cohort was profoundly delayed upon AZD0530 treatment, consistent with a driving role for up-regulated FYN kinase activity. 
Simultaneous targeting of both PTPN23 and FYN by CRISPR-CAS9 attenuated breast tumorigenesis induced by PTPN23 suppression alone

Although AZD0530 specifically targets SFKs, it shows little selectivity among the family. To address whether FYN was a primary target of AZD0530 in the above study, we took advantage of the CRISPR-CAS9 system to target simultaneously both PTPN23 and FYN in our xenograft model (Fig. 6A). For this experiment, we selected Cal51, a human TNBC (basal-like) cell line, because pathology analysis of our previous transplantation model revealed a basal-like tumor property following suppression of PTPN23. Endogenous expression of PTPN23 and FYN was detected in the Cal51 cell line (Supplemental Fig. S6A). Small guide RNAs (sgRNAs) were generated to target the catalytic domains of PTPN23 (exon 22 [E22] and exon 23 [E23]) and FYN (exon 13 [E13]) as well as AAVS1 as a negative control.

First, we established a Cal51_CAS9 cell line. After sgRNA lentiviral particle infection, GFP/mCherry marker sorting, and single clone selection, the knockout efficiency of PTPN23 and FYN in Cal51_CAS9 cells was confirmed by immunoblotting (Fig. 6B). Next, we performed in vitro cell proliferation assays using an automated cell counter with six genetically modified Cal51 cell lines over $2 \mathrm{wk}$. These cells were refreshed with growth medium plus either vehicle or $2 \mu \mathrm{M}$ AZD0530 every other day. Both PTPN23 knockout E22 and E23 cell lines proliferated faster than the CAS9 control (sgAAVS1) and FYN knockout E13 from day 4, whereas growth of the PTPN23 and FYN double-knockout cell line was slowed to the same rate as the CAS9 control (Supplemental Fig. S6B). Furthermore, treatment with $2 \mu \mathrm{M}$ AZD0530 abolished the outgrowth of PTPN23 knockout E22 and E23 cell lines, similar to the PTPN23/FYN double-knockout phenotype (Supplemental Fig. S6C). In addition, we examined the tyrosine phosphorylation status of the activation loop of SFKs in the presence and absence of AZD0530. Whereas there was a clear elevation of SFK autophosphorylation in Cal51 cells upon loss of PTPN23, this autophosphorylation was blocked up to $48 \mathrm{~h}$ after the addition of AZD0530 (Supplemental Fig. S6D,E).

Finally, in the xenograft study, we allowed 2 wk for tumor onset after Cal51 cell injection ( $n=8$ for each cohort) and then monitored tumor growth for the following $6 \mathrm{wk}$. Consistent with the previous BT474 xenograft model, we observed that tumors grew faster in PTPN23 knockout xenograft groups than those in the CAS9 control group (Fig. 6C), and codeletion of FYN in the PTPN23-deficient background significantly slowed tumor progression (Fig. $6 \mathrm{C})$. After euthanasia and necropsy, we measured tumor volume and weight for xenografts from all littermates. Consistently, the double knockout of both PTPN23 and FYN profoundly reduced both tumor volume and weight to a level similar to that of the CAS9 control and FYN knockout E13 (Fig. 6D,E). These results are further supported by the tumor dissection images in Figure 6F. Taken together, studies from CRISPR-CAS9-mediated double-knockout Cal51 cells illustrated effects on tumor progression in vivo similar to those observed following treatment with AZD0530. However, the cleaner background to this new model ameliorates concerns regarding off-target effects of the SFK inhibitor used in the previous xenograft model.

\section{Discussion}

The PTPN23 gene maps to human chromosome 3p21.3, a chromosome region with frequent loss of heterozygosity and large homozygous deletions in various epithelial cancers, including breast cancer (Toyooka et al. 2000). Consequently, it has been considered as a candidate tumor suppressor gene. It is broadly expressed in various tissues, particularly in epithelial cells (Gingras et al. 2009a). Suppression of PTPN23 promoted cell motility and invasion (Lin et al. 2011), whereas overexpression suppressed RAS-mediated transformation of fibroblasts (Cao et al. 1998) and impaired growth of renal cancer cells (Gingras et al. 2009b). Furthermore, the suppression of PTPN23 via miR-142-3p increases soft agar colony formation of a human testicular germ cell tumor cell line (Tanaka et al. 2013). In animal models, insertion of a $\beta$-geo cassette into the PTPN23 locus, which resulted in expression of a catalytically inactive truncated PTPN23 fusion protein containing only the BRO1-like domain, causes embryonic lethality in transgenic mice (Gingras et al. 2009a), consistent with an essential developmental function. Although homozygous loss was embryonic-lethal, hemizygous loss of PTPN23 resulted in cancer predisposition. For example, crossing Ptpn $23^{+/-}$mice into the E $\mu$-myc B-cell lymphoma model leads to accelerated onset of lymphoma and reduced survival, consistent with PTPN23 exerting a tumor-suppressive function in a haploinsufficient manner (Manteghi, et al. 2016). In contrast, several studies have used CRISPR screens or gene-trapping approaches that have identified PTPN23 as an essential gene (Blomen et al. 2015; Hart et al. 2015; Wang et al. 2015); for example, in Raji cells, a B-cell-derived Burrkit's lympho$\mathrm{ma}$, PTPN23 is required for proliferation and survival in this lymphoma, suggesting that it may be a point of vulnerability for the cancer (Wang et al. 2015). Such data reinforce the importance of recognizing PTPs as specific regulators of signaling pathways that may exert distinct positive or negative effects in different contexts.

There are precedents for individual PTPs serving as proto-oncogenes or tumor suppressors in specific cancers, depending on the modulation of particular cellular signaling pathways and involving different cellular processes. For example, SHP2, which is encoded by the Ptpn11 gene, was first identified as an oncogenic PTP because somatic gain-of-function mutations in SHP2 cause excessive activation of RAS-MAPK signaling and are associated with increased risk of juvenile myelomonocytic leukemia (JMML) and some solid tumors (Tartaglia et al. 2003, 2004; Mohi et al. 2005; Xu et al. 2005; Chan et al. 2009). On the other hand, Ptpn11 is a tumor suppressor gene in cartilage, in which loss of function is associated with metachondromatosis through alterations in FGF-induced 
A

\begin{tabular}{|c|c|c|}
8 weeks & \multicolumn{3}{c}{8 weeks } \\
DOB $\quad \begin{array}{c}\text { Tumor Onset } \\
\text { 2 weeks }\end{array}$ & $\begin{array}{c}\text { Tumor Size Monitor } \\
\mathbf{6} \text { weeks }\end{array}$ & Dissection
\end{tabular}

B

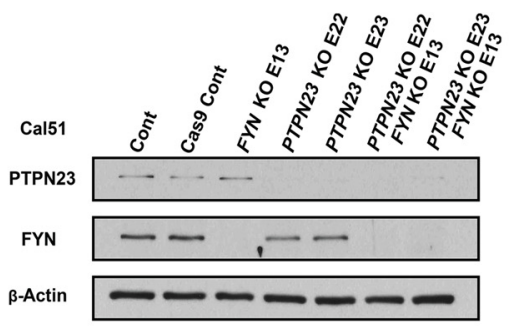

D

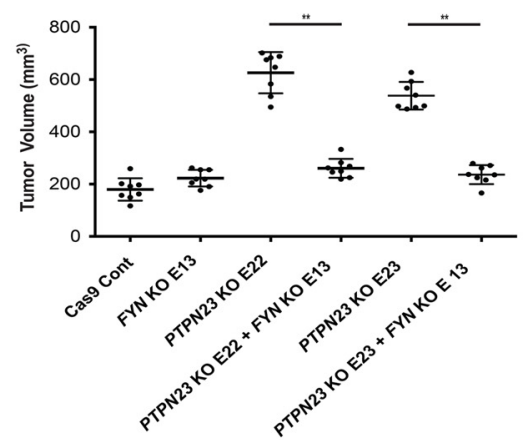

$\mathbf{F}$

Cohort 1

Left: PTPN23 KO E22 Right: Cas9 Cont

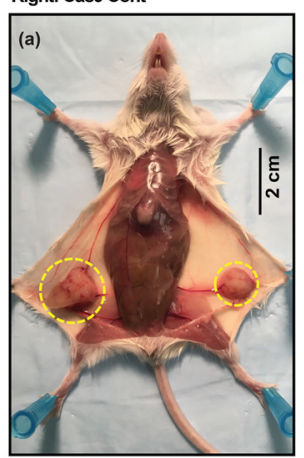

Cohort 2

Left: PTPN23 KO E23 Right: FYN KO E13

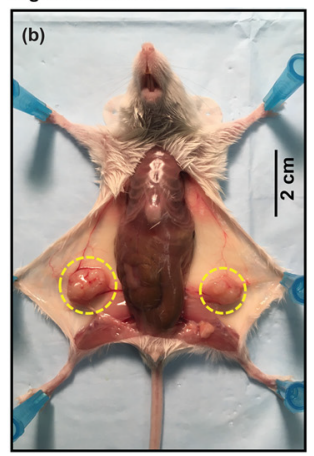

C

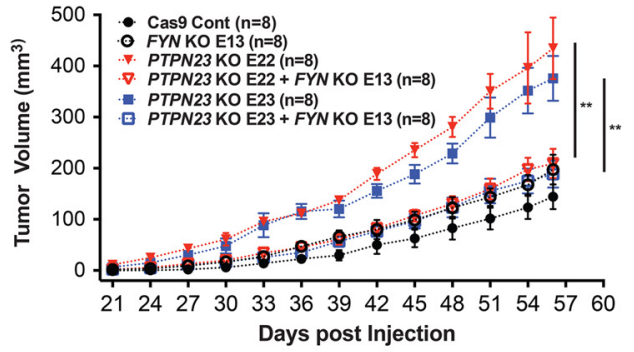

E

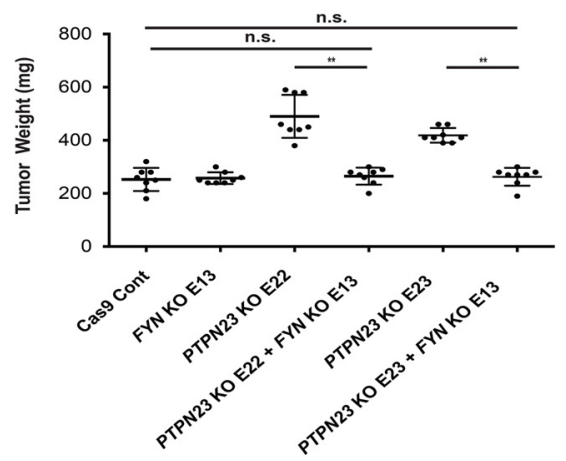

Cohort 3

Cohort 4

Left: PTPN23 KO E22 Right: PTPN23 KO E22 + FYNKO E13

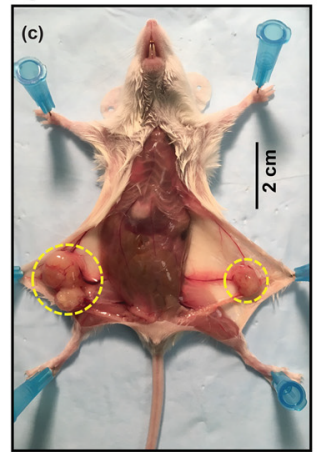

Left: PTPN23 KO E23 Right: PTPN23 KOE23 + FYNKO E13

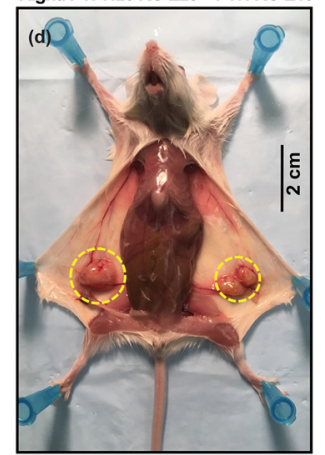

Figure 6. Simultaneous targeting of both PTPN23 and FYN by CRISPR-CAS9 attenuated breast tumorigenesis induced by PTPN23 suppression alone. $(A)$ Time line and schematic plan for the mammary gland orthotopic xenograft model using SCID/Beige mice and Cal51 cells after PTPN23 knockout, FYN knockout, and PTPN23/FYN double knockouts. (B) Prior to the xenograft experiment, the efficiency of CRISPR/CAS9 ablation using sgRNA against E22 or E23 of PTPN23 (PTPN23 KO E22 and E23) and sgRNA against E13 of FYN (FYN KO E13) was confirmed by immunoblotting with the indicated antibodies in Cal51 cell lines. An AAVS1 sgRNA expression cassette integrated into the Cal51_CAS9 cell line was also selected as a control. $\beta$-Actin was probed as the loading control. $(C)$ Four cohorts of mice $(n=8)$ were orthotopically injected with $1.5 \times 10^{6} \mathrm{Cal} 51$ cells into the abdominal mammary glands, as indicated (left/right from ventral view). The length and width of each xenograft tumor were measured with calipers every $3 \mathrm{~d}$ after injection. The tumor volume was calculated in cubic millimeters [volume $=($ width $\times$ width $\times$ length $) / 2$ ] and plotted for each of the indicated subgroups as the mean \pm SD from day 21 post-xenograft. $\left.{ }^{* *}\right) P<0.01$, two-tailed log-rank (Mantel-Cox) test. $(D, E)$ Tumors from six subgroups were collected after animal euthanasia. $(D)$ The mean \pm SD of tumor volume for each of the six indicated subgroups was plotted. Furthermore, the net weight for each tumor was measured in milligrams. $(E)$ The mean \pm SD of tumor weight for each of the six indicated subgroups was plotted. (n.s.) Not significant; $\left({ }^{* *}\right) P<0.01$, two-tailed Mann-Whitney test. $(F)$ Representative images of one xenograft animal from cohort 1 (panel $a$ ), cohort $2($ panel $b$ ), cohort 3 (panel $c$ ), and cohort 4 (panel $d$ ) illustrating the tumor in SCID/Beige mice. Bar, $2 \mathrm{~cm}$. 


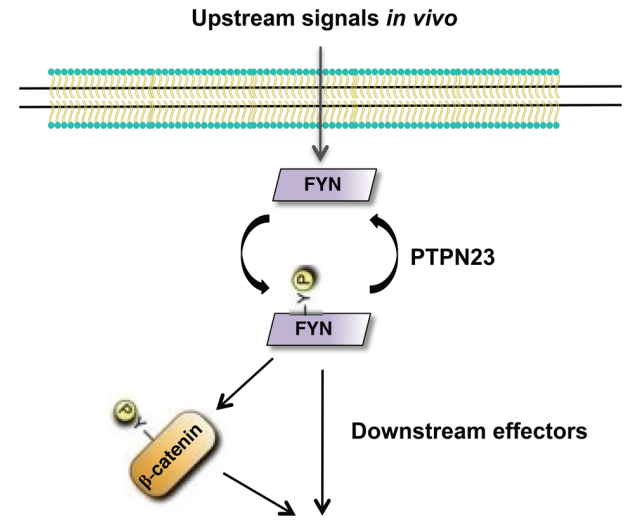

CELL TRANSFORMATION/ TUMORIGENESIS

Figure 7. Model to illustrate our proposed mechanism for breast tumorigenesis induced by suppression of PTPN23 in vivo. In this working model, the suppression of PTPN23 impaired the dephosphorylation of FYN on Y420 in cancer cells. Enhanced autophosphorylation of the tyrosine in the activation loop of FYN promoted kinase activity, which contributed to cell transformation in vivo, involving aberrant phosphorylation of substrates such as $\beta$-catenin and disrupted regulation of downstream signal cascades.

MAPK signaling (Yang et al. 2013). In addition, hepatocyte-specific ablation of the Ptpn11 gene in mice induced spontaneous macroscopic adenomas with elevated inflammatory signaling through hyperphosphorylation and activation of STAT3. Deletion of the Ptpn11 gene also significantly enhanced hepatocellular carcinoma development upon diethylnitrosamine induction, suggesting a tumor-suppressive function of SHP2 (Bard-Chapeau et al. 2011). An additional example is PTP1B. The PTPN1 gene, which encodes PTP1B, is located at chromosome $20 \mathrm{q} 13$, which is a region that is frequently amplified in breast cancer and associated with poor prognosis. In fact, PTP1B plays a positive role in promoting signaling events associated with HER2-induced breast tumorigenesis (Tonks and Muthuswamy 2007). The mechanisms underlying this effect have been proposed to involve regulation of the tyrosine phosphorylation of p62DOK and thereby control over the levels of p120RASGAP (Julien et al. 2007) as well as regulation of nonmitochondrial oxygen consumption to control tumor survival in hypoxia (Banh et al. 2016). In contrast, ablation of PTP1B in p53-null mice promoted lymphomagenesis and decreased survival rates (Dube et al. 2005). Paradoxical effects of PTP1B in tumor progression also occurred in different prostate cancer animal models. PTP1B has been linked with a tumorpromoting function for migration and invasion in prostate cancer, which is dependent on androgen receptor signaling (Lessard et al. 2012), whereas coordinated loss of PTP1B in PTEN-deficient mice caused an invasive prostate cancer upon high-fat diet feeding due to elevated PI3K-AKT signaling (Labbe et al. 2016). Finally, the PRLs (phosphatase of regenerating liver) offer an intriguing example of a PTP that serves an oncogenic function through a mechanism that does not involve the catalytic activity of the enzyme; instead, it promotes tumor growth by forming a heterodimer with members of the CNNM family of magnesium transporters and regulating levels of intracellular magnesium (Hardy et al. 2015). In contrast, Prl-3 has also been identified as a p53-inducible gene that can function positively or negatively in regulation of the cell cycle, depending on its level of expression (Basak et al. 2008). In addition, PRL-1 was shown to function as a suppressor of growth in transgenic Drosophila through a mechanism that involved suppression of SRC function (Pagarigan et al. 2013). These examples illustrate that to understand the function of specific PTPs in cancer, it is essential to identify their physiological substrates and the relevant signaling pathways that they regulate.

Our analysis established a positive correlation between the overall breast cancer survival rate and the levels of PTPN23 mRNA. Additionally, we detected lower expression of PTPN23 in several types of breast tumor compared with adjacent normal tissue (Fig. 1). Our study also showed that suppression of PTPN23 induced breast tumorigenesis in mice. The majority of tumors derived from transplanted PTPN23-deficient CD cells in vivo were typical basal-like breast cancers, adenosquamous carcinomas, or anaplastic carcinomas (Fig. 1). Using the Curtis model of 10 subtypes (Curtis et al. 2012), we assigned the effects of hemizygous or homozygous loss of PTPN23 (deletion of chromosome 3p21.3) to IntClust10. Breast cancer in this category was highly genomically instable. Most tumors were basal-like, which is in agreement with what we observed in our transplantation model. In addition, using Lehmann's study of TNBC (Lehmann et al. 2011; Lehmann and Pietenpol 2014), we assessed loss or suppression of PTPN23 as most likely to lead to breast cancer of the MSL (mesenchymal stemlike) subtype. This subtype is enriched for genes associated with growth and motility pathways and responds to dasatinib, an inhibitor of SRC/ABL PTKs. In a parallel xenograft model using a HER2-positive breast cancer cell line, suppression of PTPN23 also facilitated BT474 xenograft outgrowth (Supplemental Fig. S4), suggesting that PTPN23 may exert a general tumor-suppressive function in different subsets of breast cancer. But what of its mechanism of action?

Members of the PTP family are defined by the presence of a conserved catalytic domain. In the 37 classical pTyrspecific PTPs, we identified 10 sequence motifs that serve either structural or enzymatic functions (Andersen et al. 2001); within the PTPN23 gene, three of these motifs diverge from the consensus, leading to suggestions that PTPN23 may actually be a pseudophosphatase (Gingras et al. 2009b). One study reported that the investigators had failed to detect activity in the recombinant protein using DiFMUP (6,8-difluoro-4-methylumbelliferyl phosphate, a small-molecule substrate that can be used to detect phosphatase activity based on changes in fluorescence) and various phosphatidylinositol phospholipids (Gingras et al. 2009b). It was also reported that the recombinant catalytic domain of PTPN23 did not register activity against a panel of 38 phosphopeptides (Barr et al. 2009). In contrast, the 
active site Cys residue (and therefore, presumably, catalytic function) was required for PTPN23 to suppress RAS-mediated cell transformation (Cao et al. 1998). Similarly, in our study, we identified FYN as a physiological substrate of PTPN23, with dephosphorylation of Tyr420, the activating autophosphorylation site, underlying its tumor suppressor function in breast cancer models. The deviations from the consensus sequence of active PTP domains in PTPN23 include a substitution of His for Tyr at the position of the Tyr residue that defines the depth of the active site cleft, a substitution of Glu for Asp at the position of the residue that serves as a general acid/base in catalysis, and a substitution of Ser for Ala in the PTP signature sequence that forms the base of the active site cleft. Although these changes would be expected to impair activity, it is not clear whether they would be expected to inactivate the enzyme completely. In fact, our data illustrate that although PTPN23 does display intrinsic activity against FYN, the activity of a triple mutant (H1223Y/E1357D/S1394A) to convert to the consensus sequence was enhanced. By using the substratetrapping technology developed in the laboratory (Flint et al. 1997), we demonstrated an interaction between FYN and substrate-trapping mutant forms of PTPN23but not the wild-type form of the enzyme-that required the presence of the Tyr420 autophosphorylation site in FYN, which is indicative of an enzyme-substrate interaction. The reason for the discrepancy between these various studies is unclear; however, it is interesting to note parallels with the study of other proteins that were originally labeled pseudoenzymes. For example, within the kinome, CASK (Mukherjee et al. 2008), the JH2 domain of JAK2 (Ungureanu et al. 2011), kinase suppressor of RAS (KSR) (Brennan et al. 2011), and HER3 (Shi et al. 2010) were all described initially as pseudokinases but are now recognized as displaying intrinsic catalytic activity. This is true also for other enzyme classes; for example, UTY, a methyl lysyl demethylase (Walport et al. 2014). Thus, as individual enzymes are studied in greater depth, new insights are revealed, such as the identification of specific substrates that may lead to reassessment of initial classifications of a protein as a pseudoenzyme based primarily on sequence.

In this study, we identified FYN as a direct substrate and downstream effector of the tumor suppressor function of PTPN23 in breast tumorigenesis (Fig. 7). We validated this novel mechanism in two xenograft models by demonstrating that suppression of FYN function either with the small-molecule SFK inhibitor AZD0530 or by genetic deletion of FYN abrogated tumor formation caused by loss of PTPN23 (Figs. 5, 6). FYN is one of the 11 members of the extended SRC family of PTKs, which are the largest nonreceptor kinase family in the human genome and act as central mediators, interacting with multiple receptors and transmembrane molecules to transmit extracellular stimuli in different signaling pathways (Kim et al. 2009). SRC, the first defined oncogene, has been implicated in mediating effects of receptor tyrosine kinases such as HER2 and EGFR in breast cancer (Tan et al. 2005). Although somatic mutations in the SRC family of PTKs are rare in cancer, their overexpression or aberrant activation has been implicated in oncogenesis and metastasis of several cancers, including breast cancer. It is interesting to note that members of the PTP family have the potential to play an important role as both positive and negative regulators of SFK activity. This is based on the regulation of SFKs by their phosphorylation at two sites: an inhibitory C-terminal site of phosphorylation by the inhibitory kinase CSK and an activating site of autophosphorylation (Okada 2012). Dephosphorylation of the C-terminal inhibitory phosphorylation site, with the accompanying activation of SFKs, is a major mechanism by which PTPs may act positively to promote tyrosine phosphorylationdependent signaling. For example, CD45 (Mustelin et al. 1992), PTPa (Bhandari et al. 1998; Ponniah et al. 1999), and LAR (Tsujikawa et al. 2002) have been reported to activate FYN through dephosphorylation of the C-terminal inhibitory tyrosine residue (Tyr531). In contrast, dephosphorylation of the activating autophosphorylation site represents a mechanism for suppression of SFK activity. For example, the striatal-enriched phosphatase (STEP) has been shown to be a negative regulator of FYN activity through direct dephosphorylation of the autophosphorylated tyrosine (Tyr420) (Nguyen et al. 2002; Xu et al. 2015). Also, it has been reported that DEP-1 may partially function by inhibiting the activity of FYN to promote migration and phagocytic activity of microglial cells in the brain (Schneble et al. 2017). In contrast, PTPN23 is expressed more broadly in fibroblasts and epithelial cells of different organs (Gingras et al. 2009a). The mechanism underlying the apparent specificity of PTPN23 for FYN in this study remains to be defined. The basis for substrate specificity among the PTPs is complex and may differ from enzyme to enzyme. It seems to be more complicated than the situation in kinases, which recognize consensus primary sequence motifs surrounding the cognate phosphorylation site. It is important to note that the activation loops of the SFKs are highly conserved, indicating elements beyond the primary sequence that control PTP specificity. In fact, the expression pattern of a particular PTP may determine in part its ability to function as a regulator of SFKs in a particular cellular or tissue context.

FYN is a ubiquitously expressed SFK and acts as a major regulator of various signaling pathways that determine cell proliferation, migration, and invasion, including $\mathrm{T}$ cell receptor (TCR) signaling and neuroplasticity. Overexpression of FYN results in anchorage-independent and morphological transformation of NIH 3T3 cells (Kawakami et al. 1988). In addition, FYN promotes breast cancer metastasis via regulation of matrix rigidity at the secondary (bone) site (Kostic et al. 2009), and elevated levels of FYN have been reported in the epithelial-to-mesenchymal transition of breast cancer cells cocultured with mesenchymal stem cells (Martin et al. 2010). Aberrant expression and activation of FYN has also been implicated in several malignancies, including glioblastoma, melanoma, and prostate cancer (Huang et al. 2003; Lu et al. 2009; Posadas et al. 2009; Saito et al. 2010). In breast cancer patients, several studies have shown a strong association of elevated expression/activation of FYN, usually 
accompanied by up-regulation of FAK and c-Met and poor survival (Garcia et al. 2007; Charpin et al. 2009; Wang et al. 2010), all of which reinforce the potential significance of FYN as a therapeutic target.

Considering the critical function of SFKs in the regulation of key pathways of cell survival and proliferation, clinical trials are under way in breast cancer with SRC kinase-directed small-molecule inhibitors (dasatinib and bosutinib) in monotherapy and combination therapy (Kim et al. 2009; Mayer and Krop 2010). The transplantation animal models used in our study have now revealed a general tumor-suppressive function of PTPN23 in breast tumorigenesis and that inactivation of this PTP was associated with aberrant activation of FYN, which supports the identification of FYN as a novel therapeutic target for breast cancers defined by hemizygous or homozygous loss of PTPN23. Therefore, the search for drug-like selective inhibitors of the kinase activity of FYN may provide a new approach to treatment of breast tumors that feature loss of PTPN23 function.

\section{Materials and methods}

Cell culture

We followed published conditions for culture of CD cells (Deugnier et al. 2006), BT474 cells (Lin et al. 2011), and TNBC cell lines Cal51, MDA-MB-231, and MDA-MB-468 (Rajaram et al. 2013). HEK293T cells were maintained in growth medium containing high-glucose DMEM (Gibco, Invitrogen) supplied with 10\% FBS (Gibco, Invitrogen) and 1\% penicillin-streptomycin (Gibco, Invitrogen).

\section{RNAi}

For stable suppression of murine PTPN23 in Comma 1D cells, we expressed the pMLP retroviral vector with either of two shRNA targeting sequences against Ptpn23 (Supplemental Table S1) and sorted stable cell lines using GFP-based flow cytometry. Detailed protocols were described by Lin et al. (2011). For stable suppression of human PTPN23 and expression of firefly luciferase in BT474, we coexpressed pMLP retroviral vector with two shRNA targeting sequences against PTPN23 (Supplemental Table S1) and the pMSCV vector with a luciferase cassette. BT474 stable cell lines were selected using puromycin and hygromycin. Specifically, retroviral particles expressing shPTPN23 were generated in the 293T Phoenix cell ampho strain by cotransfecting pMLP, pCLeco, and pVSVG at a ratio of 3:1:1. Retroviral particles expressing firefly luciferase were generated following the same protocols with the pMSCV vector. Twenty-four hours after transfection, viral particles were harvested, filtered, and titered. BT474 cells were then incubated with retroviral particles in the presence of $8 \mu \mathrm{g} / \mathrm{mL}$ polybrene (Millipore). After $48 \mathrm{~h}$, BT474 cells were cultured in growth medium with $2 \mu \mathrm{g} / \mathrm{mL}$ puromycin (Sigma) and $250 \mu \mathrm{g} / \mathrm{mL}$ hygromycin (Sigma) for dual selection. The effectiveness of retroviral infection in both Comma 1D and BT474 was confirmed by immunoblotting with the indicated antibody.

\section{CRISPR/CAS9 gene knockout}

For Ptpn23 and/or Fyn gene ablation in Cal51 cells, we established a Cal51 cell line with stable expression of hSpcas9 (Cal51_CAS9).
We expressed a pLKO lentiviral vector with an hSpcas9 cassette and selected the stable cell line using blasticidin. For CRISPR knockout of PTPN23 in Cal51, we transfected Cal51_CAS9 with a LRG lentiviral vector expressing sgRNA sequences against PTPN23 and sorted stable cell lines using GFP-based flow cytometry. Specifically, lentiviral particles expressing sgPTPN23 were generated in HEK293T cells by cotransfecting sgRNA vector, pPAX2, and pVSVG at a ratio of 4:3:2. Twenty-four hours after transfection, viral particles were harvested, filtered, and titered. Cal51_CAS9 cells were then incubated with lentiviral particles in the presence of $8 \mu \mathrm{g} / \mathrm{mL}$ polybrene (Millipore). After $48 \mathrm{~h}$, Cal51 cells were sorted according to GFP expression followed by single clone selection. For CRISPR knockout of FYN in Cal51, we followed the same protocols with LRCh vector and mCherry-based flow cytometry sorting. For double knockouts of PTPN23 and FYN in Cal51, we cotransfected Cal51_CAS9 with both LRG and LRCh lentiviral vectors for sgRNA against PTPN23 and FYN, respectively. Stable cell lines were sorted using dual-color (GFP and mCherry) flow cytometry. The sgRNA sequences used for PTPN23 and FYN knockout are listed in Supplemental Table S1. The effectiveness of CRISPR gene knockout was further confirmed by immunoblotting with the indicated antibody.

\section{Animal work}

All study protocols involving mice were approved by the Institutional Animal Care and Use Committee of Cold Spring Harbor Laboratory and conducted in accordance with National Institutes of Health guidelines for the care and use of animals. In the orthotopic transplantation model, CD cells were trypsinized, counted, and resuspended at a concentration of 100,000 cells per $10 \mu \mathrm{L}$ in RPMI within two passages after sorting. Three-week-old BALB/c mice (Charles River Laboratories) were anesthetized, and the fourth (left) and ninth (right) inguinal mammary fat pads were cleared of endogenous epithelium by removing the tissue between the nipple and the lymph node according to established procedures (Ehmann et al. 1987). Next, 100,000 cells in $10 \mu \mathrm{L}$ RPMI were injected into the epithelium-free fat pad, and the incision was closed with sutures. Mice were monitored by palpation twice a week for tumor onset from 1 mo after mammary epithelial cell inoculation. Mice were euthanized when tumors reached $1.5-2 \mathrm{~cm}$ in diameter or $56 \mathrm{wk}$ after transplantation. All tumors and mammary glands were harvested and weighed before they were fixed for histological analysis and frozen for future biochemical analysis.

In BT474 xenograft models, $1.5 \times 10^{6}$ luciferase-expressing BT474 cells were injected orthotopically into mammary fat pads of SCID-Beige mice (Tectonic Laboratory) in $15 \mu \mathrm{L}$ of a 1:1 mixture with DMEM and growth factor-reduced Matrigel (BD Biosciences). In each case, shPTPN23 BT474 cells were injected into one side of the abdominal fat pad, and shCont cells were injected contralaterally. Therefore, each mouse was injected with tumor cells on one side and control cells on the other. In the pilot study, animals were allowed to develop tumors. Tumor growth was monitored periodically by injecting $100 \mu \mathrm{L}$ of $15 \mathrm{mg} / \mathrm{kg} \mathrm{D-}$ luciferin (Gold Biotechnology) intraperitoneally and imaging the animal using a Xenogen imager (Xenogen IVIS-200 optical in vivo imaging system). Tumor volume (in cubic millimeters) was measured with calipers before imaging $($ calculated volume $=$ [width $\times$ width $\times$ length] $/ 2$ ). In the rescue study, animals injected with BT474 cells (shCont and shPTPN23 \#1058) were randomly assigned into two groups to receive DMSO/saline (vehicle) or 25 $\mathrm{mg} / \mathrm{kg}$ AZD0530 (LC Laboratories) treatment orally every third day. In addition to caliper measurements, tumor growth was monitored by bioluminescence imaging. Mice were euthanized 
when tumors reached $1.5-2 \mathrm{~cm}$ in diameter or at the end point of the study. All tumors were harvested and weighed before they were fixed for histological analysis and frozen for future biochemical analysis. The same procedures were used for the Cal51 xenograft model; $1.5 \times 10^{6} \mathrm{Cal} 51$ cells were injected orthotopically into mammary fat pads of SCID-Beige mice (Tectonic Laboratory), and tumor growth in the four cohorts of animals was monitored as described above.

\section{Histology}

Paraffin-embedded tissues were sectioned and stained with H\&E or stored for further paraffin or fluorescence IHC. All slides were digitally scanned with an Aperio ScanScope XT automated scanning system. Images were shared in a Cold Spring Harbor Laboratory public database, and the pathological stage and histological grade of CD-derived breast tumors were analyzed with the help of J.E. Wilkinson.

\section{Fluorescence microscopy}

For GFP detection of dissected tissues from transplantation animals, tumors or mammary glands were fixed in $4 \%$ paraformaldehyde overnight after dissection. Next, tissues were embedded in $3 \%$ agarose and cut into $50-\mu \mathrm{m}$-thick sections using a vibratome (Leica, VT1000S). GFP fluorescence images were acquired using a Zeiss LSM 510 microscope with a 40× objective.

Cell and tissue lysate preparation, immunoprecipitation, and immunoblotting

Comma 1D, BT474, Cal51, or HEK293T cells were lysed in either $1 \%$ NP40 lysis buffer (20 mM HEPES at pH 7.5, $150 \mathrm{mM} \mathrm{NaCl}$, $1 \% \mathrm{NP} 40,50 \mathrm{mM} \mathrm{NaF}, 1 \mathrm{mM} \mathrm{Na}_{3} \mathrm{VO}_{4}, 10 \%$ glycerol, protease inhibitor cocktail from Roche) or modified RIPA buffer $(50 \mathrm{mM}$ Tris-Cl at $\mathrm{pH} 7.4,150 \mathrm{mM} \mathrm{NaCl}, 1 \% \mathrm{NP} 40,1 \%$ sodium deoxycholate, $0.1 \%$ SDS, $50 \mathrm{mM} \mathrm{NaF}, 1 \mathrm{mM} \mathrm{Na} \mathrm{VO}_{4}, 10 \%$ glycerol, protease inhibitor cocktail from Roche) for $30 \mathrm{~min}$ at $4^{\circ} \mathrm{C}$. Cell debris was removed by centrifugation. For mammary glands and tumors, extra connective tissue was carefully dissected from harvested tissues. Tissue samples were ground into a powder under liquid nitrogen and lysed for $30 \mathrm{~min}$ on ice in $1 \%$ NP40 lysis buffer. The tissue lysates were cleared by centrifugation at $12,000 \mathrm{~g}$ for $15 \mathrm{~min}$ at $4^{\circ} \mathrm{C}$. Protein concentration was determined by Bradford protein assay (Bio-Rad).

For immunoprecipitation, cell or tissue extracts were incubated with the indicated antibody for $4 \mathrm{~h}$ in a cold room with rotation followed by further incubation for $1 \mathrm{~h}$ after addition of $1: 1$ protein A/G agarose beads (GE Healthcare). Immunoprecipitates were washed with lysis buffer three times before gel electrophoresis. For immunoblotting, proteins were resolved by SDS-PAGE and transferred onto nitrocellulose membranes. The membrane was incubated overnight at $4^{\circ} \mathrm{C}$ with primary antibody followed by incubation with the horseradish peroxidase-conjugated secondary antibody. Immunoreactive proteins were detected by chemiluminescence (Thermo Fisher). The primary antibodies used for immunoprecipitation and immunoblotting were as follows: PTPN23 (human, Proteintech; mouse, polyclonal antibody from Dr. Pause, McGill University, Montreal, Canada); pTyr142 $\beta$-catenin and FYN (Abcam); $\beta$-catenin, auto-pTyr SFK (pTyr416 SFK), c-ter pTyr SFK (pTyr527 SFK), SRC, FER, c-MET, and YES (Cell Signaling Technology); and $\beta$-actin (Sigma).

\section{Site-directed mutagenesis}

All point mutations were individually introduced into the coding sequences of the specific target gene regardless of vectors by using a QuickChange site-directed mutagenesis kit (Stratagene) to generate mutant forms of full-length PTPN23 (C1329S and E1357A), the PTPN23 catalytic domain (C1329S, H1223Y, E1357D, S1394A, and H1223Y/S1394A/E1357D), and full-length FYN (Y420F and Y531F).

Recombinant protein purification and phosphatase activity assay

All pET-28a constructs with a PTPN23 catalytic domain-coding cassette insert were introduced into the bacterial strain BL21RIL for recombinant protein production and purification. The proteins were purified following published procedures (Lin et al. 2011). After purification, protein concentration was measured by extinction coefficient at A280 (Nanodrop 1000, Thermo Fisher) and based on the calculated extinction coefficient. Proteins were stored frozen in $30 \%$ glycerol-containing buffer for phosphatase activity assay. Plasmid expressing the FYN Y531F mutant was transfected into Comma 1D cells for $24 \mathrm{~h}$ followed by immunoprecipitation from cell lysates with an antibody against FYN. Each immunoprecipitate was split into equal aliquots and incubated with purified PTPN23 and its various mutants (range $0-200 \mathrm{ng}$ ) for $30 \mathrm{~min}$ at $30^{\circ} \mathrm{C}$. The in vitro dephosphorylation reaction was terminated with Laemmli sample buffer, and proteins were separated by SDS-PAGE. Substrate dephosphorylation was visualized by immunoblotting.

\section{Cell proliferation assay}

CellTiter-Glo luminescence cell viability assay (Promega) was used to evaluate the effect of ablating PTPN23 on proliferation of CD or human breast cancer cells (BT474 and Cal51). In brief, 1000 cells per well were seeded in a 96-well plate and grown for the indicated time intervals. CellTiter-Glo reagent was added to each well and mixed for $15 \mathrm{~min}$ on an orbital shaker to induce cell lysis, followed by luminescence reading. Results represent mean \pm SEM from three independent experiments.

\section{IHC and TMA}

Human breast cancer TMAs (TMA BC081116c and TMA BR10011a) were purchased from US Biomax, Inc. Immunohistochemical staining for PTPN23, pTyr142 $\beta$-catenin, $\beta$-catenin, and auto-pTyr SFK (pY416 SFK) was performed in Histology Shared Resource at Cold Spring Harbor Laboratory (DAB chromogen kit, Vector Laboratories). Slides were digitally scanned with the Aperio ScanScope software. For quantification of IHC staining, the Aperio cytoplasm analysis algorithm was used according to the manual. For analysis, $10-15$ areas containing only tumor or adjacent normal epithelial cells were traced for analysis. Primary antibodies used for IHC and TMA were as follows: PTPN23 (human, Proteintech; mouse, polyclonal antibody from Dr. Pause, McGill University, Montreal, Canada), pTyr142 $\beta$-catenin (Abcam), auto pTyr SFK (pTyr416 SFK, R\&D Systems), and $\beta$-catenin (Cell Signaling Technology).

\section{Clinical data analysis for PTPN23 in breast cancer}

For analysis of PTPN23 in breast cancer clinical samples, a data set of 1069 breast cancer cases (BRCA) was extracted from TCGA database (https://cancergenome.nih.gov); clinical followup information is in Supplemental Table S2. All cases were divided into high and low PTPN23 subgroups based on the median 
PTPN23 mRNA expression value. Kaplan-Meier survival curves for both the low and high PTPN23 subgroups were generated using the survival package in $\mathrm{R}$ (https://cran.r-project.org/ package=survival). For analysis of PTPRG, we followed the same protocols with the same data set of breast cancer cases. Statistical significance was assessed by the log-rank test.

\section{Statistics}

The breast cancer clinical data were presented as Kaplan-Meier survival curves, generated with the survival package in R, and statistical comparison of the curves was based on the log-rank test. All other charts were generated with GraphPad Prism 6.0 software. The median was chosen such that the two groups (high and low) would have an equal number of patients and thus that the statistics would not be driven by a minor (underpowered) population. In the orthotopic transplantation model, comparisons of Kaplan-Meier survival and tumor-free curves were also based on the log-rank test. In xenograft models with BT474 as well as Cal51 cells, statistical comparisons of animal body weight and in vivo tumor volume between different cohorts were based on the log-rank test. Statistical comparisons of ex vivo tumor volume and tumor weight were based on the MannWhitney test. A two-tailed Student's $t$-test was also used for comparisons of data from in vitro cell proliferation assays as well as IHC and TMA assays. Statistical significance was defined as $P<0.05\left(^{*}\right)$ and $P<0.01\left(^{* *}\right)$ for all tests.

\section{Acknowledgments}

We thank Mikala Egeblad (Cold Spring Harbor Laboratory, NY) for the Cal51 and MDA-MB-468 cell lines, Christopher Vakoc (Cold Spring Harbor Laboratory, NY) for the CAS9 expression construct and sgRNA expression constructs LRG (Lenti_sgRNA_EFS_GFP) and LRCh (Lenti_sgRNA_EFS_mCherry), Arnim Pause (McGill University, Montreal, Canada) for the polycolonal PTPN23 antibody, and Senthil K. Muthuswamy (Harvard University, MA) for sharing the CD cell line as well as for many helpful discussions. This work was performed with assistance from Cold Spring Harbor Laboratory Shared Resources, especially support from the Animal Shared Resource and Flow Cytometry Shared Resource. This work was supported by National Institutes of Health grant CA53840 to N.K.T., and Cold Spring Harbor Laboratory Cancer Centre Support Grant CA45508. N.K.T. is also grateful for support from the Gladowsky Breast Cancer Foundation, the Don Monti Memorial Research Foundation, the Irving Hansen Foundation, the Estate of Thomas C. Nelson, and the Robertson Research Fund. S.Z. and N.K.T. designed the experiments. S.Z. contributed to all aspects of the experimental work. G.F. contributed to the RNAi screen and provided insightful thoughts and discussion. Y.H. and M.H. contributed to the bioinformatics analysis. J.E.W. contributed to the pathology studies. All of the authors contributed to the analysis of the data. S.Z. and N.K.T. wrote the paper. N.K.T. directed the study.

\section{References}

Ali N, Zhang L, Taylor S, Mironov A, Urbe S, Woodman P. 2013. Recruitment of UBPY and ESCRT exchange drive HD-PTPdependent sorting of EGFR to the MVB. Curr Biol 23: 453-461.

Alonso A, Sasin J, Bottini N, Friedberg I, Osterman A, Godzik A, Hunter T, Dixon J, Mustelin T. 2004. Protein tyrosine phosphatases in the human genome. Cell 117: 699-711.
Andersen JN, Mortensen OH, Peters GH, Drake PG, Iversen LF, Olsen OH, Jansen PG, Andersen HS, Tonks NK, Moller NP. 2001. Structural and evolutionary relationships among protein tyrosine phosphatase domains. Mol Cell Biol 21: 7117-7136.

Andersen JN, Jansen PG, Echwald SM, Mortensen OH, Fukada T, Del Vecchio R, Tonks NK, Moller NP. 2004. A genomic perspective on protein tyrosine phosphatases: gene structure, pseudogenes, and genetic disease linkage. FASEB J 18: 8-30.

Angeloni D. 2007. Molecular analysis of deletions in human chromosome 3p21 and the role of resident cancer genes in disease. Brief Funct Genomic Proteomic 6: 19-39.

Ardini E, Agresti R, Tagliabue E, Greco M, Aiello P, Yang LT, Menard S, Sap J. 2000. Expression of protein tyrosine phosphatase a (RPTPa) in human breast cancer correlates with low tumor grade, and inhibits tumor cell growth in vitro and in vivo. Oncogene 19: 4979-4987.

Banh RS, Iorio C, Marcotte R, Xu Y, Cojocari D, Rahman AA, Pawling J, Zhang W, Sinha A, Rose CM, et al. 2016. PTP1B controls non-mitochondrial oxygen consumption by regulating RNF213 to promote tumour survival during hypoxia. Nat Cell Biol 18: 803-813.

Bard-Chapeau EA, Li S, Ding J, Zhang SS, Zhu HH, Princen F, Fang DD, Han T, Bailly-Maitre B, Poli V, et al. 2011. Ptpn11/ Shp2 acts as a tumor suppressor in hepatocellular carcinogenesis. Cancer Cell 19: 629-639.

Barr AJ, Ugochukwu E, Lee WH, King ON, Filippakopoulos $\mathrm{P}$, Alfano I, Savitsky P, Burgess-Brown NA, Muller S, Knapp S. 2009. Large-scale structural analysis of the classical human protein tyrosine phosphatome. Cell 136: 352-363.

Basak S, Jacobs SB, Krieg AJ, Pathak N, Zeng Q, Kaldis P, Giaccia AJ, Attardi LD. 2008. The metastasis-associated gene Prl-3 is a p53 target involved in cell-cycle regulation. Mol Cell 30: 303-314.

Bentires-Alj M, Gil SG, Chan R, Wang ZC, Wang Y, Imanaka N, Harris LN, Richardson A, Neel BG, Gu H. 2006. A role for the scaffolding adapter GAB2 in breast cancer. Nat Med 12: 114-121.

Bhandari V, Lim KL, Pallen CJ. 1998. Physical and functional interactions between receptor-like protein-tyrosine phosphatase $\alpha$ and p59fyn. J Biol Chem 273: 8691-8698.

Bissig C, Gruenberg J. 2014. ALIX and the multivesicular endosome: ALIX in Wonderland. Trends Cell Biol 24: 19-25.

Blomen VA, Majek P, Jae LT, Bigenzahn JW, Nieuwenhuis J, Staring J, Sacco R, van Diemen FR, Olk N, Stukalov A, et al. 2015. Gene essentiality and synthetic lethality in haploid human cells. Science 350: 1092-1096.

Brennan DF, Dar AC, Hertz NT, Chao WC, Burlingame AL, Shokat KM, Barford D. 2011. A Raf-induced allosteric transition of KSR stimulates phosphorylation of MEK. Nature 472: 366-369.

Cao L, Zhang L, Ruiz-Lozano P, Yang Q, Chien KR, Graham RM, Zhou M. 1998. A novel putative protein-tyrosine phosphatase contains a BRO1-like domain and suppresses Ha-ras-mediated transformation. J Biol Chem 273: 21077-21083.

Chan G, Kalaitzidis D, Usenko T, Kutok JL, Yang W, Mohi MG, Neel BG. 2009. Leukemogenic Ptpn11 causes fatal myeloproliferative disorder via cell-autonomous effects on multiple stages of hematopoiesis. Blood 113: 4414-4424.

Charpin C, Secq V, Giusiano S, Carpentier S, Andrac L, Lavaut MN, Allasia C, Bonnier P, Garcia S. 2009. A signature predictive of disease outcome in breast carcinomas, identified by quantitative immunocytochemical assays. Int J Cancer 124: 2124-2134. 
Curtis C, Shah SP, Chin SF, Turashvili G, Rueda OM, Dunning MJ, Speed D, Lynch AG, Samarajiwa S, Yuan Y, et al. 2012. The genomic and transcriptomic architecture of 2,000 breast tumours reveals novel subgroups. Nature 486: 346-352.

Danielson KG, Oborn CJ, Durban EM, Butel JS, Medina D. 1984. Epithelial mouse mammary cell line exhibiting normal morphogenesis in vivo and functional differentiation in vitro. Proc Natl Acad Sci 81: 3756-3760.

Deugnier MA, Faraldo MM, Teuliere J, Thiery JP, Medina D, Glukhova MA. 2006. Isolation of mouse mammary epithelial progenitor cells with basal characteristics from the Comma-D $\beta$ cell line. Dev Biol 293: 414-425.

Dube N, Bourdeau A, Heinonen KM, Cheng A, Loy AL, Tremblay ML. 2005. Genetic ablation of protein tyrosine phosphatase $1 \mathrm{~B}$ accelerates lymphomagenesis of p53-null mice through the regulation of B-cell development. Cancer Res 65: 1008810095.

Ehmann UK, Guzman RC, Osborn RC, Young JT, Cardiff RD, Nandi S. 1987. Cultured mouse mammary epithelial cells: normal phenotype after implantation. J Natl Cancer Inst 78: 751-757.

Flint AJ, Tiganis T, Barford D, Tonks NK. 1997. Development of 'substrate-trapping' mutants to identify physiological substrates of protein tyrosine phosphatases. Proc Natl Acad Sci 94: $1680-1685$.

Freiss G, Vignon F. 2004. Protein tyrosine phosphatases and breast cancer. Crit Rev Oncol Hematol 52: 9-17.

Garcia S, Dales JP, Charafe-Jauffret E, Carpentier-Meunier S, Andrac-Meyer L, Jacquemier J, Andonian C, Lavaut MN, Allasia C, Bonnier P, et al. 2007. Overexpression of c-Met and of the transducers PI3K, FAK and JAK in breast carcinomas correlates with shorter survival and neoangiogenesis. Int J Oncol 31: 49-58.

Gil-Henn H, Elson A. 2003. Tyrosine phosphatase- $\varepsilon$ activates Src and supports the transformed phenotype of Neu-induced mammary tumor cells. J Biol Chem 278: 15579-15586.

Gingras MC, Kharitidi D, Chenard V, Uetani N, Bouchard M, Tremblay ML, Pause A. 2009a. Expression analysis and essential role of the putative tyrosine phosphatase His-domain-containing protein tyrosine phosphatase (HD-PTP). Int J Dev Biol 53: 1069-1074.

Gingras MC, Zhang YL, Kharitidi D, Barr AJ, Knapp S, Tremblay ML, Pause A. 2009b. HD-PTP is a catalytically inactive tyrosine phosphatase due to a conserved divergence in its phosphatase domain. PLoS One 4: e5105.

Hardy S, Uetani N, Wong N, Kostantin E, Labbe DP, Begin LR, Mes-Masson A, Miranda-Saavedra D, Tremblay ML. 2015. The protein tyrosine phosphatase PRL-2 interacts with the magnesium transporter CNNM3 to promote oncogenesis. Oncogene 34: 986-995.

Harris IS, Blaser H, Moreno J, Treloar AE, Gorrini C, Sasaki M, Mason JM, Knobbe CB, Rufini A, Halle M, et al. 2014. PTPN12 promotes resistance to oxidative stress and supports tumorigenesis by regulating FOXO signaling. Oncogene 33: 1047-1054.

Hart T, Chandrashekhar M, Aregger M, Steinhart Z, Brown KR, MacLeod G, Mis M, Zimmermann M, Fradet-Turcotte A, Sun S, et al. 2015. High-resolution CRISPR screens reveal fitness genes and genotype-specific cancer liabilities. Cell 163: $1515-1526$.

Hennequin LF, Allen J, Breed J, Curwen J, Fennell M, Green TP, Lambert-van der Brempt C, Morgentin R, Norman RA, Olivier A, et al. 2006. N-(5-chloro-1,3-benzodioxol-4-yl)-7-[2-(4-methylpiperazin-1-yl)ethoxy]-5-(tetrahydro-2H-pyran-4-yloxy)quinazolin-4-amine, a novel, highly selective, orally available, dual-specific c-Src/Abl kinase inhibitor. I Med Chem 49: 6465-6488.

Hochgrafe F, Zhang L, O'Toole SA, Browne BC, Pinese M, Porta Cubas A, Lehrbach GM, Croucher DR, Rickwood D, Boulghouriian A, et al. 2010. Tyrosine phosphorylation profiling reveals the signaling network characteristics of Basal breast cancer cells. Cancer Res 70: 9391-9401.

Huang J, Asawa T, Takato T, Sakai R. 2003. Cooperative roles of Fyn and cortactin in cell migration of metastatic murine melanoma. J Biol Chem 278: 48367-48376.

Hunter T. 2009. Tyrosine phosphorylation: thirty years and counting. Curr Opin Cell Biol 21: 140-146.

Ibarra I, Erlich Y, Muthuswamy SK, Sachidanandam R, Hannon GJ. 2007. A role for microRNAs in maintenance of mouse mammary epithelial progenitor cells. Genes Dev 21:3238-3243.

Julien SG, Dube N, Read M, Penney J, Paquet M, Han Y, Kennedy BP, Muller WJ, Tremblay ML. 2007. Protein tyrosine phosphatase 1B deficiency or inhibition delays ErbB2-induced mammary tumorigenesis and protects from lung metastasis. Nat Genet 39: 338-346.

Julien SG, Dube N, Hardy S, Tremblay ML. 2011. Inside the human cancer tyrosine phosphatome. Nat Rev Cancer 11:35-49.

Kawakami T, Kawakami Y, Aaronson SA, Robbins KC. 1988. Acquisition of transforming properties by FYN, a normal SRC-related human gene. Proc Natl Acad Sci 85: 3870-3874.

Kharitidi D, Apaja PM, Manteghi S, Suzuki K, Malitskaya E, Roldan A, Gingras MC, Takagi J, Lukacs GL, Pause A. 2015. Interplay of endosomal $\mathrm{pH}$ and ligand occupancy in integrin a 51 ubiquitination, endocytic sorting, and cell migration. Cell Rep 13: 599-609.

Kim LC, Song L, Haura EB. 2009. Src kinases as therapeutic targets for cancer. Nat Rev Clin Oncol 6: 587-595.

Kostic A, Lynch CD, Sheetz MP. 2009. Differential matrix rigidity response in breast cancer cell lines correlates with the tissue tropism. PLoS One 4: e6361.

Labbe DP, Uetani N, Vinette V, Lessard L, Aubry I, Migon E, Sirois J, Haigh JJ, Begin LR, Trotman LC, et al. 2016. PTP1B deficiency enables the ability of a high-fat diet to drive the invasive character of PTEN-deficient prostate cancers. Cancer Res 76: 3130-3135.

Lehmann BD, Pietenpol JA. 2014. Identification and use of biomarkers in treatment strategies for triple-negative breast cancer subtypes. J Pathol 232: 142-150.

Lehmann BD, Bauer JA, Chen X, Sanders ME, Chakravarthy AB, Shyr Y, Pietenpol JA. 2011. Identification of human triplenegative breast cancer subtypes and preclinical models for selection of targeted therapies. J Clin Invest 121: 2750-2767.

Lessard L, Labbe DP, Deblois G, Begin LR, Hardy S, Mes-Masson AM, Saad F, Trotman LC, Giguere V, Tremblay ML. 2012. $\mathrm{PTP} 1 \mathrm{~B}$ is an androgen receptor-regulated phosphatase that promotes the progression of prostate cancer. Cancer Res 72: 1529-1537.

Levea CM, McGary CT, Symons JR, Mooney RA. 2000. PTP LAR expression compared to prognostic indices in metastatic and non-metastatic breast cancer. Breast Cancer Res Treat 64: 221-228.

Lin G, Aranda V, Muthuswamy SK, Tonks NK. 2011. Identification of PTPN23 as a novel regulator of cell invasion in mammary epithelial cells from a loss-of-function screen of the 'PTP-ome'. Genes Dev 25: 1412-1425.

Lu KV, Zhu S, Cvrljevic A, Huang TT, Sarkaria S, Ahkavan D, Dang J, Dinca EB, Plaisier SB, Oderberg I, et al. 2009. Fyn and SRC are effectors of oncogenic epidermal growth factor receptor signaling in glioblastoma patients. Cancer Res 69: 6889-6898. 
Manteghi S, Gingras MC, Kharitidi D, Galarneau L, Marques M, Yan M, Cencic R, Robert F, Paquet M, Witcher M, et al. 2016. Haploinsufficiency of the ESCRT component HD-PTP predisposes to cancer. Cell Rep 15: 1893-1900.

Martin FT, Dwyer RM, Kelly J, Khan S, Murphy JM, Curran C, Miller N, Hennessy E, Dockery P, Barry FP, et al. 2010. Potential role of mesenchymal stem cells (MSCs) in the breast tumour microenvironment: stimulation of epithelial to mesenchymal transition (EMT). Breast Cancer Res Treat 124: 317-326.

Mayer EL, Krop IE. 2010. Advances in targeting SRC in the treatment of breast cancer and other solid malignancies. Clin Cancer Res 16: 3526-3532.

Mohi MG, Williams IR, Dearolf CR, Chan G, Kutok JL, Cohen S, Morgan K, Boulton C, Shigematsu H, Keilhack H, et al. 2005. Prognostic, therapeutic, and mechanistic implications of a mouse model of leukemia evoked by Shp2 (PTPN11) mutations. Cancer Cell 7: 179-191.

Mukherjee K, Sharma M, Urlaub H, Bourenkov GP, Jahn R, Sudhof TC, Wahl MC. 2008. CASK functions as a $\mathrm{Mg}^{2+}$-independent neurexin kinase. Cell 133: 328-339.

Mustelin T, Pessa-Morikawa T, Autero M, Gassmann M, Andersson LC, Gahmberg CG, Burn P. 1992. Regulation of the p59fyn protein tyrosine kinase by the CD45 phosphotyrosine phosphatase. Eur I Immunol 22: 1173-1178.

Nguyen TH, Liu J, Lombroso PJ. 2002. Striatal enriched phosphatase 61 dephosphorylates Fyn at phosphotyrosine 420. J Biol Chem 277: 24274-24279.

Okada M. 2012. Regulation of the SRC family kinases by Csk. Int J Biol Sci 8: 1385-1397.

Ostman A, Hellberg C, Bohmer FD. 2006. Protein-tyrosine phosphatases and cancer. Nat Rev Cancer 6: 307-320.

Pagarigan KT, Bunn BW, Goodchild J, Rahe TK, Weis JF, Saucedo LJ. 2013. Drosophila PRL-1 is a growth inhibitor that counteracts the function of the Src oncogene. PLoS One 8: e61084.

Piedra J, Miravet S, Castano J, Palmer HG, Heisterkamp N, Garcia de Herreros A, Dunach M. 2003. p120 Catenin-associated Fer and Fyn tyrosine kinases regulate $\beta$-catenin Tyr-142 phosphorylation and $\beta$-catenin-a-catenin Interaction. Mol Cell Biol 23: 2287-2297.

Ponniah S, Wang DZ, Lim KL, Pallen CJ. 1999. Targeted disruption of the tyrosine phosphatase PTPa leads to constitutive downregulation of the kinases Src and Fyn. Curr Biol 9: $535-538$

Posadas EM, Al-Ahmadie H, Robinson VL, Jagadeeswaran R, Otto K, Kasza KE, Tretiakov M, Siddiqui J, Pienta KJ, Stadler WM, et al. 2009. FYN is overexpressed in human prostate cancer. BJU Int 103: 171-177.

Radke I, Gotte M, Kersting C, Mattsson B, Kiesel L, Wulfing P. 2006. Expression and prognostic impact of the protein tyrosine phosphatases PRL-1, PRL-2, and PRL-3 in breast cancer. Br J Cancer 95: 347-354.

Rajaram M, Li J, Egeblad M, Powers RS. 2013. System-wide analysis reveals a complex network of tumor-fibroblast interactions involved in tumorigenicity. PLOS Genet 9: e1003789.

Rakha EA, Reis-Filho JS, Ellis IO. 2008. Basal-like breast cancer: a critical review. J Clin Oncol 26: 2568-2581.

Ramesh M, Krishnan N, Muthuswamy SK, Tonks NK. 2015. A novel phosphatidic acid-protein-tyrosine phosphatase D2 axis is essential for ERBB2 signaling in mammary epithelial cells. J Biol Chem 290: 9646-9659.

Saito YD, Jensen AR, Salgia R, Posadas EM. 2010. Fyn: a novel molecular target in cancer. Cancer 116: 1629-1637.

Schneble N, Muller J, Kliche S, Bauer R, Wetzker R, Bohmer FD, Wang ZQ, Muller JP. 2017. The protein-tyrosine phosphatase
DEP-1 promotes migration and phagocytic activity of microglial cells in part through negative regulation of fyn tyrosine kinase. Glia 65: 416-428.

Senchenko VN, Liu J, Loginov W, Bazov I, Angeloni D, Seryogin Y, Ermilova V, Kazubskaya T, Garkavtseva R, Zabarovska VI, et al. 2004. Discovery of frequent homozygous deletions in chromosome 3p21.3 LUCA and AP20 regions in renal, lung and breast carcinomas. Oncogene 23: 5719-5728.

Shi F, Telesco SE, Liu Y, Radhakrishnan R, Lemmon MA. 2010. ErbB3/HER3 intracellular domain is competent to bind ATP and catalyze autophosphorylation. Proc Natl Acad Sci 107: 7692-7697.

Sliwkowski MX, Mellman I. 2013. Antibody therapeutics in cancer. Science 341: 1192-1198.

Sun T, Aceto N, Meerbrey KL, Kessler JD, Zhou C, Migliaccio I, Nguyen DX, Pavlova NN, Botero M, Huang J, et al. 2011. Activation of multiple proto-oncogenic tyrosine kinases in breast cancer via loss of the PTPN12 phosphatase. Cell 144: 703-718.

Tan M, Li P, Klos KS, Lu J, Lan KH, Nagata Y, Fang D, Jing T, Yu D. 2005. ErbB2 promotes Src synthesis and stability: novel mechanisms of Src activation that confer breast cancer metastasis. Cancer Res 65: 1858-1867.

Tanaka K, Kondo K, Kitajima K, Muraoka M, Nozawa A, Hara T. 2013. Tumor-suppressive function of protein-tyrosine phosphatase non-receptor type 23 in testicular germ cell tumors is lost upon overexpression of miR142-3p microRNA. J Biol Chem 288: 23990-23999.

Tartaglia M, Niemeyer CM, Fragale A, Song X, Buechner J, Jung A, Hahlen K, Hasle H, Licht JD, Gelb BD. 2003. Somatic mutations in PTPN11 in juvenile myelomonocytic leukemia, myelodysplastic syndromes and acute myeloid leukemia. Nat Genet 34: 148-150.

Tartaglia M, Martinelli S, Cazzaniga G, Cordeddu V, Iavarone I, Spinelli M, Palmi C, Carta C, Pession A, Arico M, et al. 2004. Genetic evidence for lineage-related and differentiation stage-related contribution of somatic PTPN11 mutations to leukemogenesis in childhood acute leukemia. Blood 104: 307-313.

Tonks NK, Muthuswamy SK. 2007. A brake becomes an accelerator: PTP1B - a new therapeutic target for breast cancer. Cancer Cell 11: 214-216.

Toyooka S, Ouchida M, Jitsumori Y, Tsukuda K, Sakai A, Nakamura A, Shimizu N, Shimizu K. 2000. HD-PTP: a novel protein tyrosine phosphatase gene on human chromosome 3p21.3. Biochem Biophys Res Commun 278: 671-678.

Tsujikawa K, Ichijo T, Moriyama K, Tadotsu N, Sakamoto K, Sakane N, Fukada S, Furukawa T, Saito H, Yamamoto H. 2002. Regulation of Lck and Fyn tyrosine kinase activities by transmembrane protein tyrosine phosphatase leukocyte common antigen-related molecule. Mol Cancer Res 1: $155-163$.

Ungureanu D, Wu J, Pekkala T, Niranjan Y, Young C, Jensen ON, Xu CF, Neubert TA, Skoda RC, Hubbard SR, et al. 2011. The pseudokinase domain of JAK2 is a dual-specificity protein kinase that negatively regulates cytokine signaling. Nat Struct Mol Biol 18: 971-976.

Walport LJ, Hopkinson RJ, Vollmar M, Madden SK, Gileadi C, Oppermann U, Schofield CJ, Johansson C. 2014. Human $\mathrm{UTY}(\mathrm{KDM} 6 \mathrm{C})$ is a male-specific $N^{\varepsilon}$-methyl lysyl demethylase. J Biol Chem 289: 18302-18313.

Wang X, Fredericksen ZS, Vierkant RA, Kosel ML, Pankratz VS, Cerhan JR, Justenhoven C, Brauch H, Olson JE, Couch FJ. 2010. Association of genetic variation in mitotic kinases 
with breast cancer risk. Breast Cancer Res Treat 119: 453-462.

Wang T, Birsoy K, Hughes NW, Krupczak KM, Post Y, Wei JJ, Lander ES, Sabatini DM. 2015. Identification and characterization of essential genes in the human genome. Science 350: 1096-1101.

Xu R, Yu Y, Zheng S, Zhao X, Dong Q, He Z, Liang Y, Lu Q, Fang $\mathrm{Y}, \mathrm{Gan} \mathrm{X}$, et al. 2005. Overexpression of Shp2 tyrosine phosphatase is implicated in leukemogenesis in adult human leukemia. Blood 106: 3142-3149.

Xu J, Kurup P, Foscue E, Lombroso PJ. 2015. Striatal-enriched protein tyrosine phosphatase regulates the PTPa/Fyn signaling pathway. J Neurochem 134: 629-641.

Yang W, Wang J, Moore DC, Liang H, Dooner M, Wu Q, Terek R, Chen Q, Ehrlich MG, Quesenberry PJ, et al. 2013. Ptpn11 deletion in a novel progenitor causes metachondromatosis by inducing hedgehog signalling. Nature 499: 491-495.

Yu M, Lin G, Arshadi N, Kalatskaya I, Xue B, Haider S, Nguyen F, Boutros PC, Elson A, Muthuswamy LB, et al. 2012. Expression profiling during mammary epithelial cell three-dimensional morphogenesis identifies PTPRO as a novel regulator of morphogenesis and ErbB2-mediated transformation. Mol Cell Biol 32: 3913-3924.

Yuan T, Wang Y, Zhao ZJ, Gu H. 2010. Protein-tyrosine phosphatase PTPN9 negatively regulates ErbB2 and epidermal growth factor receptor signaling in breast cancer cells. I Biol Chem 285: $14861-14870$.

Zhao S, Sedwick D, Wang Z. 2015. Genetic alterations of protein tyrosine phosphatases in human cancers. Oncogene 34: 3885-3894.

Zheng J, Kulp SK, Zhang Y, Sugimoto Y, Dayton MA, Govindan MV, Brueggemeier RW, Lin YC. 2000. 17 $\beta$-estradiol-regulated expression of protein tyrosine phosphatase $\gamma$ gene in cultured human normal breast and breast cancer cells. Anticancer Res 20: $11-19$.

Zhu JH, Chen R, Yi W, Cantin GT, Fearns C, Yang Y, Yates JR III, Lee JD. 2008. Protein tyrosine phosphatase PTPN13 negatively regulates Her2/ErbB2 malignant signaling. Oncogene 27: 2525-2531. 


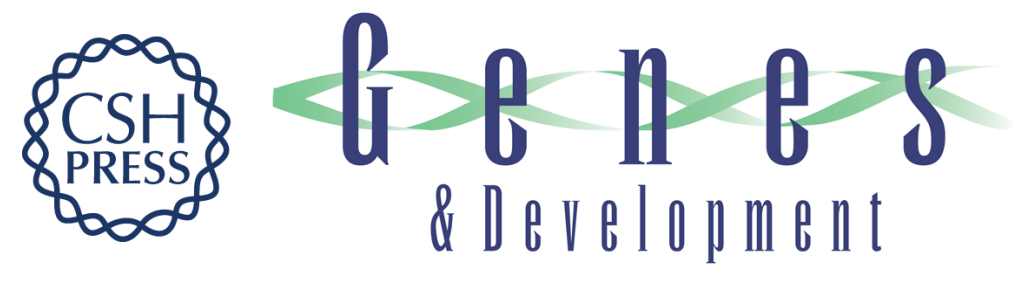

\section{Suppression of protein tyrosine phosphatase N23 predisposes to breast tumorigenesis via activation of FYN kinase}

Siwei Zhang, Gaofeng Fan, Yuan Hao, et al.

Genes Dev. 2017, 31: originally published online October 24, 2017

Access the most recent version at doi:10.1101/gad.304261.117

\section{Supplemental http://genesdev.cshlp.org/content/suppl/2017/10/24/gad.304261.117.DC1 Material}

References This article cites 92 articles, 33 of which can be accessed free at: http://genesdev.cshlp.org/content/31/19/1939.full.html\#ref-list-1

Creative This article is distributed exclusively by Cold Spring Harbor Laboratory Press for the first Commons six months after the full-issue publication date (see

License http://genesdev.cshlp.org/site/misc/terms.xhtml). After six months, it is available under a Creative Commons License (Attribution-NonCommercial 4.0 International), as described at http://creativecommons.org/licenses/by-nc/4.0/.

Email Alerting Receive free email alerts when new articles cite this article - sign up in the box at the top Service right corner of the article or click here.

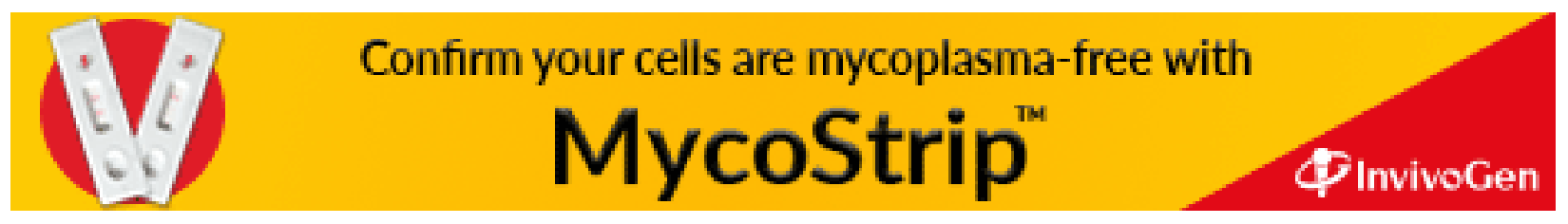

